\title{
Structure and Evolution of Nearby Stars with Planets. I. Short-Period Systems
}

\author{
Eric B. Ford, Frederic A. Rasio \\ Department of Physics, MIT 6-201, Cambridge, MA 02139 \\ eford@mit.edu; rasio@mit.edu \\ and \\ Alison Sills ${ }^{1}$ \\ Department of Astronomy, Yale University, P.O. Box 208101, New Haven, CT 06520
}

\begin{abstract}
Using the Yale stellar evolution code, we have calculated theoretical models for nearby stars with planetary-mass companions in short-period nearly circular orbits: 51 Pegasi, $\tau$ Bootis, $v$ Andromedae, $\rho^{1}$ Cancri, and $\rho$ Coronae Borealis. We present tables listing key stellar parameters such as mass, radius, age, and size of the convective envelope as a function of the observable parameters (luminosity, effective temperature, and metallicity), as well as the unknown helium fraction. For each star we construct best models based on recently published spectroscopic data and the present understanding of galactic chemical evolution. We discuss our results in the context of planet formation theory, and, in particular, tidal dissipation effects and stellar metallicity enhancements.
\end{abstract}

Subject headings: Planets and Satellites: General — Stars: Planetary Systems; Stars: individual - 51 Pegasi, $\tau$ Bootis, $v$ Andromedae, $\rho^{1}$ Cancri, $\rho$ Coronae Borealis

\section{Introduction and Motivation}

The detection of planets outside the Solar System constitutes one of the most exciting recent developments in astronomy and astrophysics. These discoveries of extrasolar planets will lead to significant improvements in our understanding of many processes related to planet and star formation, as well as deeper questions such as the existence of extraterrestrial life in the Universe. We now know of more planets outside the Solar System than inside (including planets around pulsars; Wolszczan 1994). Several groups have reported detections of Jupiter-type planets around nearby solar-like stars (see Table 1), and new announcements continue to arrive every few months. We expect that radial velocity surveys already in progress (Marcy et al. 1997; Korzennik et al. 1997; Mayor \& Queloz 1997; Cochran et al. 1997a; Butler et al. 1998) will discover many more in the near future. Other techniques such as photometry (Borucki \& Summers 1984) and astrometry (Gatewood 1996; Pravdo \& Shaklan 1996), including interferometric astrometry (Colavita \& Shao 1994) and, in the future, space-based interferometric astrometry (Unwin et al. 1996; Boden et al. 1996), may lead to additional detections. In addition, observations of protostellar disks (Mannings 1998) and T Tauri stars (Beckwith \& Sargent 1996) can provide important information

\footnotetext{
${ }^{1}$ Present Address: Ohio State University; asills@astronomy.ohio-state.edu
} 
about planet formation. In the long term, space based interferometry appears very promising (Unwin et al. 1996; Boden et al. 1996).

This paper is the first of a series in which we use the Yale Rotating Evolution Code (the YREC code developed by Demarque and collaborators; see Guenther et al. 1992) to calculate theoretical models for the present structure and past evolution of stars known to harbor planets, using recently measured stellar parameters and Hipparcos parallaxes as constraints. Our goal is to obtain a set of self-consistent theoretical models that will provide the best possible values (with realistic error estimates) for all derived stellar parameters, such as mass and radius, which are crucial for a number of theoretical investigations of these systems. In this first paper we present a general description of the stellar evolution code and our method for constructing the theoretical models.

\subsection{Tidal Dissipation}

Our models will provide a detailed description of the stellar convective zone, which is essential when calculating tidal dissipation through eddy viscosity (e.g., Zahn 1977; Zahn \& Bouchet 1989). Tidal dissipation may have played a crucial role in the formation and evolution of the 51 Peg-type systems. Despite observational biases, the clustering of orbital periods around $3-4 \mathrm{~d}$ for these systems is probably not a coincidence. The San Francisco State University (SFSU) extrasolar planet search (Marcy et al. 1997) is capable of detecting periodic radial velocity variations with an amplitude of $12 \mathrm{~m} \mathrm{~s}^{-1}$, corresponding to a $1 M_{J}$ planet orbiting at $1 \mathrm{AU}$ from a $1 M_{\odot}$ star given a favorable inclination. Additional factors, such as the variability of stellar photospheres and the longer time span of observations necessary to confirm the Keplerian nature of the radial velocity variations, can also inhibit detection of planets with larger semi-major axes. Nevertheless, the present low yield of extrasolar planets with larger semi-major axes is likely indicative of a real pattern. Periods of $3-4 \mathrm{~d}$ correspond to the onset of strong tidal dissipation in such systems. When the star is rapidly rotating, tidal torques may prevent the planet from spiraling all the way into the star and being destroyed (Lin, Bodenheimer, \& Richardson 1996; Trilling et al. 1998). This may be essential in formation scenarios invoking slow inward migration of giant planets in a protostellar disk. Alternatively, tidal dissipation effects may circularize a highly eccentric orbit produced by a dynamical interaction (Rasio \& Ford 1996). In addition, for a slowly rotating star (most of the stars in Table 1 are known to be slowly rotating today), tidal dissipation leads to (possibly rapid) orbital decay, and therefore the survival of the system to the present provides an important constraint on theoretical models (Rasio et al. 1996). In some cases this may also provide an upper limit on the companion mass (see §4.1).

Tidal dissipation is particularly interesting yet difficult to study theoretically in these systems since the tidal pumping period (half the orbital period) is short compared to the typical eddy turnover timescale in the stellar convective envelope. In this regime, the efficiency of tidal dissipation can be drastically reduced (Goldreich \& Keeley 1977), although the details of the theory in this case are rather controversial (see Goodman \& Oh 1997 and references therein). The 51 Peg-type systems therefore provide important new constraints on the theory of tidal dissipation in a regime where it is particularly poorly understood. A better understanding of tidal effects (such as binary circularization and spin synchronization) in this regime would be applicable to a number of other important systems, including pre-main-sequence (hereafter PMS) binaries (Zahn \& Bouchet 1989). The $51 \mathrm{Peg}$ system has a very short-period (4.2 d) orbit, which makes it particularly interesting in the context of tidal dissipation theory (Rasio et al. 1996; Marcy et al. 1997). 


\subsection{Planet Formation}

The properties of the new planets are surprising (see Table 1). Most are Jupiter-mass objects in very tight circular orbits or in wider eccentric orbits. The standard model for planet formation in our Solar System cannot explain their orbital properties (see, e.g., Lissauer 1993; Boss 1995). According to this standard model, planetary orbits should be nearly circular, and giant planets should be found at large distances $(\gtrsim 1 \mathrm{AU})$ from the central star, where the temperature in the protostellar nebula is low enough for icy materials to condense (Boss 1995, 1996). These simple predictions of the standard model for the formation of the Solar System are at odds with the observed parameters of most detected extrasolar planets. With only one exception (47 UMa), the new planets all come within $1 \mathrm{AU}$ of the central star. Three planets (51 Peg, $\tau$ Boo, $v$ And) are in extremely tight circular orbits with periods of only a few days. Two planets $\left(\rho^{1} \mathrm{Cnc}\right.$ and $\left.\rho \mathrm{CrB}\right)$ have circular orbits with somewhat longer periods, of order tens of days. Three companions with wider orbits (16 Cyg B, 70 Vir, and HD 114762) have very large eccentricities $(\sim 0.5)$. A number of different theoretical scenarios have been proposed to explain the unexpected orbital properties of these extrasolar planets. Our calculations will provide accurate stellar parameters that can be used to obtain better constraints on these formation scenarios.

Four specific mechanisms have been suggested as possible processes to bring a giant planet into a short-period orbit around its star. One mechanism is a secular interaction with a distant binary companion. If the orbit of a wide binary is inclined relative to a planet's orbit by more than $\sim 40^{\circ}$, the relative inclination of the binary star can couple to a secular increase in the eccentricity in the planet's orbit (Holman, Touma, \& Tremaine 1996; Mazeh, Krymolowski, \& Rosenfeld 1996). The amplitude of the eccentricity perturbation depends on the relative inclination of the orbits, but is independent of the mass of the binary companion. If the orbital planes have a very high relative inclination, then an eccentricity approaching unity can be induced. In some cases this may cause a collision with the star. However, if dissipation is significant (in the star, in the planet, or in a disk), then the orbit could circularize at a small distance. Similarly, if the primary star has a significant quadrupole moment, then tidal dissipation in the star could stop the growth of the eccentricity oscillations and drive a gradual decrease in the semi-major axis of the planet's orbit (Kiseleva \& Eggleton 1997; Eggleton \& Kiseleva 1997). This mechanism of secular perturbations from a distant binary companion can also produce significant eccentricities for the longer period extrasolar planets. It is interesting to note that four of the five planets with semi-major axes, $a \lesssim 0.25 \mathrm{AU}$ are in wide binary systems (Hoffleit \& Warren 1991). 51 Peg has been searched extensively for a binary companion (Marcy et al. 1997), but none has been found. If the relative inclination less than $\sim 40^{\circ}$, then the amplitude of the eccentricity oscillations becomes sensitive to the mass of the perturbing body, the ratio of the semi-major axes, and the eccentricity of the outer orbit. For a binary companion of stellar mass, only a small range of parameters will produce large eccentricities without disrupting the system. The large mass ratio of either star to the planet makes it easy for the system to be disrupted. Since the planet is the least massive of the three bodies by many orders of magnitude, the planet is almost certainly the body to be ejected, if the system dissociates.

Dissipation in the protostellar nebula is a second possible mechanism for forming a short period planet. Since the orbital migration of the planet would tend to accelerate with decreasing separation from the star, the dissipation has to switch off at a critical moment for a planet to end up so close to its parent star without being disrupted. Possible mechanisms for stopping the inward migration include Roche lobe overflow and tidal coupling to a rapidly rotating star (Trilling et al. 1998; Lin et al. 1996). Another possibility is that the migration stops when the planet arrives at the inner edge of a disk limited by a magnetosphere around the star (Lin et al. 1996). 
A resonant interaction with a disk of planetesimals is another possible source of orbital migration, but this requires a very large protoplanetary disk mass if a $\sim 1 M_{\mathrm{J}}$ planet is to migrate inwards all the way to $\sim 0.1 \mathrm{AU}$ (Murray et al. 1998). The advantage, however, is that the migration is halted naturally at short distances when the majority of perturbed planetesimals collide with the star rather than escaping on nearly parabolic orbits. Wide eccentric orbits can also be produced for planets more massive than $\sim 3 M_{\mathrm{J}}$. It seems that such a massive disk would be likely to produce more than one planet, and thus it is important to understand how a second or third planet would affect this scenario.

The fourth mechanism is based on dynamical instabilities in a system originally containing multiple giant planets of comparable masses (Rasio \& Ford 1996). If either the orbital radii evolve secularly at different rates (significant orbital migration is thought to have occurred in the outer Solar System; see Goldreich \& Tremaine 1980; Malhotra 1995) or if the masses increase significantly as the planets accrete their gaseous envelopes (Lissauer 1993), then the orbits could become unstable. Alternatively, if either the second or third mechanism for producing short period planets were truncated when the disk mass dwindled, they could leave two planets in dynamically unstable orbits. Similarly, a wide binary companion could drive a secular increase in the planets' eccentricity until they became dynamically unstable. In any case, the evolution can lead to a dynamical instability of the orbits and a strong gravitational interaction between two planets (Gladman 1993; Chambers, Wetherill, \& Boss 1996). This interaction can lead to the ejection of one planet, leaving the other in an eccentric orbit. If the pericenter distance of the inner planet is sufficiently small, its orbit can later circularize at an orbital separation of a few stellar radii (Rasio et al. 1996). This mechanism can produce eccentric systems in two different ways. After many encounters, one planet can be ejected from the system leaving the other planet in an eccentric orbit by itself (Rasio \& Ford 1996; Katz 1997). In this scenario it should be expected that the second planet has been ejected and is no longer in the system (Black 1997). Alternatively, if the two planets collide, they can produce a more massive planet in an eccentric orbit (Rasio \& Ford 1996; Lin \& Ida 1997). Dynamical instabilities in systems containing more than two giant planets of comparable masses have also been studied. Weidenschilling \& Marzari (1996) have obtained numerical results for the case of 3 planets, while Lin \& Ida (1997) performed simulations for systems containing up to 9 planets. In this case successive mergers between two or more planets can lead to the formation of a fairly massive $\left(\gtrsim 10 M_{\mathrm{J}}\right)$ object in a wide, eccentric orbit. While it is almost certain that this mechanism operates in many systems with multiple planets, it is not clear that it can produce a fraction of 51 Peg-like systems as large as observed. Extensive numerical simulations will be necessary to obtain good statistics on this theory (Ford \& Rasio 1998). Observational selection effects must also be better understood for a meaningful comparison with the properties of the detected systems.

All these dynamical processes can also affect the evolution of the central star. Marcy et al. (1997) and Drake et al. (1998) argue that the observed rapid rotation of $\tau$ Boo is likely caused by the tidal interaction between the star and its close planetary companion (our results do not support this interpretation; see §4.1). Several stars with short-period planets have high metallicities $\left(\left[\frac{\mathrm{Fe}}{\mathrm{H}}\right] \gtrsim 0.2\right.$; see Gonzalez 1997a, 1998ab and Table 2). Gonzalez (1998a) proposes that their metallicities have been enhanced by the accretion of high-Z material. Alternatively, the correlation could arise, if metal-rich stars have metal-rich disks which

are more likely to form planets. Thus, understanding the relationship between metallicity and the existence of short-period planets may be important in constraining the mechanisms which produce these planets. We discuss this in more detail after constructing models for these stars (See §4.2).

\section{Constructing Stellar Models}




\subsection{The Code}

We use the Yale Rotating Evolution Code in its non-rotating mode to calculate stellar models. YREC is a Henyey code which solves the equations of stellar structure in one dimension. The chemical composition of each shell is updated separately using the nuclear reaction rates of Bahcall \& Pinsonneault (1992). The initial chemical mixture is the solar mixture of Anders \& Grevesse (1989), scaled to match the metallicity of the star being modeled. For regions of the star where $\log T(K) \geq 6$, all atoms are assumed to be fully ionized. For regions where $\log T(K) \leq 5.5$, particle densities are determined by solving the Saha equation for the single ionization state of hydrogen and the metals, and for the single and double ionization states of helium. In the transition region between these two temperatures, both formulations are weighted with a ramp function and averaged. The equation of state includes both radiation pressure and electron degeneracy pressure. We use the latest OPAL opacities (Iglasias \& Rogers 1996) for the interior of the star down to temperatures of $\log T(K)=4$. For lower temperatures, we use the low-temperature opacities of Alexander $\&$ Ferguson (1994). We use an Eddington T- $\tau$ relationship for the atmosphere, and, where appropriate, the standard Böhm-Vitense mixing length theory of convection (Cox 1968; Böhm-Vitense 1958) with the ratio of the mixing length to the pressure scale height, $\alpha=1.70$. This value of $\alpha$, as well as the solar hydrogen abundance, $X_{\odot}=0.697$, was obtained by calibrating models against observations of the present day Sun using the aforementioned physics.

Astroseismology has made it possible to test models of stellar interiors directly. Models of the Sun (see, e.g., Guenther et al. 1992) are constantly being refined with newer and more sophisticated input physics, including diffusion of helium and heavy elements (Guenther \& Demarque 1997) and detailed hydrodynamic calculations of the surface convection zone (Demarque et al. 1997). YREC has also been used to calculate models of other single field stars. Most recently, observations of p-mode oscillations in $\eta$ Bootis, a nearby subgiant, prompted detailed stellar modeling of that star (Guenther \& Demarque 1996). This work forms the basis of our method. The modeled frequency spectra of the Sun and $\eta$ Bootis agree quite well with observations. YREC has also been used to model Procyon (Guenther \& Demarque 1993) and both components of the binary star $\alpha$ Centauri (Edmonds et al. 1992). Although stellar models have also predicted seismology spectra for Procyon and $\alpha$ Centauri, no convincing observations of the frequency spectra have yet been made.

In constructing stellar models a variety of formalisms are available for the treatment of the equation of state, the stellar atmosphere, and convection. We adopted formalisms which result in a good solar model, and are standard enough to be applicable to all low-mass stars. We have neglected non-standard effects such as diffusion of helium and heavy elements, rotation, and magnetic fields since these processes do not significantly change the overall properties of low-mass stars like the sun. Solar models constructed by YREC with the set of standard formalisms adopted here reproduce the observed solar p-mode spectrum to within 1\% (Guenther et al. 1992). The incorporation of newer physics can reduce this difference by a few tenths of a percent.

We have chosen to use the Saha equation of state rather than MHD (Mihalas et al. 1988) or OPAL (Rogers \& Iglesias 1994). The MHD and OPAL equations of state are very similar to each other, and differ from the YREC implementation of the Saha equation by less than 1\%. The OPAL equation of state results in a slightly deeper convection zone for the sun, and a worse fit to the observed p-mode spectrum (Guenther et al. 1996) compared to the Saha equation of state.

For the treatment of convective zones we have adopted the standard Böhm-Vitense mixing length theory. Some other theories, such as that of Canuto \& Mazzitelli (1992), produce solar models which have 
p-mode spectra in slightly better agreement with observations to the observed spectrum. Currently, a number of groups are using 3-D numerical simulations of turbulent convection to develop a more realistic description of stellar convective zones (e.g. Kim \& Chan 1998). However, the results of these simulations are not yet available in sufficient detail to be incorporated into general stellar models.

The mixing length is chosen so that our solar model reproduces the solar luminosity and solar radius at the solar age. Our best value of the mixing length parameter (ratio of mixing length to the local pressure scale height) is 1.7. To test the effect of the mixing length on the stellar parameters, for several of our best models we varied the mixing length from 1.4 to 2.0 (a large range). We found that the luminosity of the star typically varied by $\sim 3 \%$, the radius by $\sim 5 \%$, the mass of the convective envelope by $\sim 35 \%$, and the radius of the convective envelope by $\sim 2 \%$. We have also performed a number of tests to check that the choice of atmosphere model does not affect our results significantly. For examples, we find that using the Kurucz tabulated atmospheres (Kurucz 1991) rather than the Eddington T- $\tau$ relation increases the calculated effective temperatures and increases the calculated radii by less than $1 \%$. We can test our complete set of physical parameters and assumptions by comparing the calculated depth of the solar convection zone to that derived from the observed solar p-mode spectrum. Christensen-Dalsgaard et al. (1991) give the radius at the base of the solar convection zone to be $0.713 \pm 0.003 \mathrm{R}_{\odot}$. Our calibrated model produces a convection zone which begins at $0.647 \mathrm{R}_{\odot}$, a difference of $\sim 10 \%$. In summary, we expect that the calculated stellar

radii and temperatures will be accurate to within about $5 \%$, and the calculated size of the convection zones will be accurate to within $20 \%$, including all possible changes to the physics of the models.

\subsection{Iteration Method}

We constructed models of $0.6 M_{\odot}, 0.8 M_{\odot}, 1.0 M_{\odot}, 1.2 M_{\odot}, 1.4 M_{\odot}$, and $1.6 M_{\odot}$ PMS stars by solving the Lane-Emden equation for a polytrope of index $n=1.5$. YREC evolved the models to the zero-age main sequence (hereafter ZAMS). These ZAMS models serve as the starting point for all our subsequent modeling.

For each model we construct, we first specify a mass and metallicity and then evolve a ZAMS model in an attempt to match a given effective temperature $T_{\text {eff }}$ and luminosity $L$. First, YREC chooses a ZAMS model with a similar mass and metallicity and scales the ZAMS model to match the desired values of $M_{*}$ and $Z$ (indirectly affecting $X$ and $Y$ ). The scaled ZAMS model is numerically relaxed before YREC begins to evolve the model to the desired radius (determined from effective temperature and luminosity). YREC iterates the above procedure, returning to the ZAMS model, but rescaling to new values $X$ and $Y$ so as to improve the match to the desired luminosity and radius.

Thus, a given run fits a model to the desired luminosity and radius (and hence temperature). For such a run we hold $M_{*}$ and $Z$ constant, explicitly vary $Y$, and let $X$, the age, and the other stellar parameters vary as a result of the changing composition. This procedure is repeated for several masses yielding a set of models which share the same specified metallicity, luminosity, and temperature, but differ in mass, age, composition, and other parameters.

Since we vary the helium content in our models, it is possible to obtain models that match the observed parameters but have unrealistic compositions. To select our final "best"' model, we consider the ratio

$$
\frac{\delta Y}{\delta Z} \equiv \frac{Y-Y_{\odot}}{Z-Z_{\odot}}
$$

where $Y$ and $Z$ are the helium and heavy element abundances of our model. Based on studies of galactic 
evolution, including H II regions (Pagel et al. 1992) and low-metallicity blue compact galaxies (Izotov et al. 1997), we impose the canonical constraint $\frac{\delta Y}{\delta Z}=2.5 \pm 1$ (Bressan et al. 1994; Edvardsson et al. 1993). For our "best" model, we interpolate to find a mass and age corresponding to $\frac{\delta Y}{\delta Z}=2.5$ for the specified parameters. Similarly, we present models for $\frac{\delta Y}{\delta Z}$ equal to 1.5 and 3.5 from which we determine theoretical error bars given a set of assumed observational parameters. Due to the physical scatter and the possibility of systematic errors in the determination of $\frac{\delta Y}{\delta Z}$, we also include models for $\frac{\delta Y}{\delta Z}$ equal to 5.0 and 0.0 . The latter may also be useful for comparisons with studies in which $Y=Y_{\odot}$ is assumed. Fortunately, the theoretical uncertainties derived from the variation of $\frac{\delta Y}{\delta Z}$ are, in general, smaller than the uncertainties in the stellar observations, as will be discussed in the next section. The above procedure is repeated for each set of observed parameters, $Z, T_{\text {eff }}$, and $L$. For some sets of observed parameters, YREC is unable to converge on a single self-consistent model.

\subsection{Observational Data}

The above procedure for constructing a set of models to match a real star requires three basic input parameters from observations. The luminosity, $L$, effective temperature, $T_{\text {eff }}$, and metallicity, $Z$, must be known accurately. The observational uncertainty in these quantities limits the accuracy with which we can derive the values of other stellar parameters. As more observational data become available, these can be used to constrain our models more tightly.

\subsubsection{Luminosities}

To minimize systematic errors, we consistently use the stellar luminosities and parallaxes obtained from the Hipparcos catalog. The recent release of the Hipparcos data (ESA 1997, Perryman et al. 1997) is extremely useful for this type of study, since accurate distances to all the relevant stars are now available. The Hipparcos parallax data have greatly improved astronomical distances and tightly constrained luminosities, nearly removing an entire degree of freedom from the models. Since only the brightest (and therefore the closest) solar-type stars are targets for present spectroscopic surveys, all the solar-type stars presently known to have planets are nearby and have distances directly determined by Hipparcos parallax data. This distance is combined with the Hipparcos apparent visual magnitude to obtain a visual luminosity.

One minor complication is the application of bolometric corrections to convert visual luminosities to integrated luminosities. We estimate bolometric corrections by interpolating in $[\mathrm{Fe} / \mathrm{H}], \log g$, and $T_{\text {eff }}$ across color calibration grids used for the Yale Isochrones (Green et al. 1987). This method of estimating bolometric corrections does introduce a dependence of our results on $\log g$. However, bolometric corrections depend only weakly on $\log g$, and the uncertainty in $\log g$ hardly introduces any uncertainty in the bolometric correction. Comparing our bolometric corrections with other tables indicates that there is a $\sim 5 \%$ systematic uncertainty in our luminosities due to the potential systematic error in the bolometric corrections. From our full set of models we see that the uncertainty in the integrated luminosity is normally insignificant compared to other sources of error. 


\subsubsection{Spectroscopic Data}

In addition to the luminosity, our models also requires a knowledge of each star's metallicity and effective temperature. Effective temperatures and metallicities can be obtained from either photometric or spectroscopic data, although the latter are normally more accurate. In this paper we have chosen values for the necessary parameters from the literature. To minimize systematic errors, we decided to use temperatures, metallicities, and surface gravities obtained from a single source, the recent spectroscopic observations of all the solar-like stars with planets by Gonzalez (1997ab, 1998ab). His determinations of $T_{\text {eff }}, \log g,[\mathrm{Fe} / \mathrm{H}]$, and the microturbulence parameter $\zeta_{t}$, are from self-consistent iterative solutions which matched high resolution spectra of Fe I and Fe II lines to Kurucz (1993) model atmospheres. Gonzalez (1998) quotes typical errors of $75 \mathrm{~K}, 0.06$, and 0.05 for $T_{\text {eff }},[\mathrm{Fe} / \mathrm{H}]$, and $\log g$, respectively. These formal errors may be somewhat smaller than the actual uncertainty in the values, but the general agreement with other observations is reassuring. We construct additional models when other observations significantly deviate from the Gonzalez results, or when we cannot construct models simultaneously matching all the observed parameters. We summarize the observed parameters of stars with planets in Table 2. We will list other determinations of the stellar parameters, $T_{\mathrm{eff}},[\mathrm{Fe} / \mathrm{H}], \log g$, and $v \sin i$, as we discuss each star individually.

\subsubsection{Other Observational Parameters}

Several other stellar parameters can be measured observationally. For example, $\log g$ is routinely measured spectroscopically. While determinations of $\log g$ serve as a useful tool for purposes such as determining bolometric corrections (see $\S 2.3 .1)$, present determinations $\left(\sigma_{\log g(\operatorname{cgs})} \sim 0.1\right)$ provide only loose constraints $(\sim 25 \%)$ on $M_{*} / R_{*}^{2}$. Thus, we use measurements of $\log g$ merely as a consistency check for our models.

Analysis of Ca II H and $\mathrm{K}$ emission can be used to detect the stellar rotation period. When a rotation period is not detected, it can be predicted using an empirical relation between the Ca II flux and the rotation period (Noyes et al. 1984). The rotation period can be combined with the radius to yield an equatorial velocity, $v_{\text {eq }}$. This can be compared with the observed $v \sin i$ to yield the inclination angle between the star's equator and the line of sight. If the angle between the star's equator and the planet's orbital plane is presumed small, one can determine the planet's actual mass, $m$, from its minimum mass, $m \sin i$. Unfortunately, $v \sin i$ is very difficult to determine observationally, as it is delicately coupled to the macroscopic turbulence parameter. We list only recent determinations of $v \sin i$ and take even these somewhat cautiously. Empirically, both the level of Ca II H and K activity and the stellar rotation frequency have been found to decrease with age and this relationship can be used to estimate the age of the star (Baliunas et al. 1995; Soderblom et al. 1991). We will compare our determinations of the age with those predicted by the activity-age relation.

Photometric determinations of the angular diameter are possible with the Barnes and Evans relationship (Barnes, Evans, \& Moffet 1978; Moffett \& Barnes 1979) or the more recent infrared flux method (Blackwell et al. 1990). Combined with parallax measurements, these yield a stellar radius. Given the relatively high uncertainties associated with these methods, we also use these observations only as a consistency check. 


\section{Results}

For each star we have computed a grid of models surrounding the observed values of $L, T_{\text {eff }}$, and $[\mathrm{Fe} / \mathrm{H}]$. As more observations become available, it is hoped that our large grids of models will allow observers to translate their values of the observed parameters into physical parameters such as mass, radius, age, and size of the convective zone. In general, interpolating across grids of stellar models can be inaccurate as non-linearities become significant. However, we have calculated a fine enough grid of models for each star such that interpolation should yield reasonable results. The set of models printed here is only a small subset of our large grid of models. Here we present models corresponding the adopted values of $T_{\text {eff }},[\mathrm{Fe} / \mathrm{H}]$, and $L$ and models corresponding to a $1-\sigma$ uncertainty in any one of these parameters. For each model we include the mass $M_{*}$, the age, the mass of the convective envelope $M_{\mathrm{ce}}$, the radius of the convective envelope $R_{\mathrm{ce}}$, the pressure scale height at the base of the convective envelope PSH, the radius $R_{*}$, and the surface gravity $\log g$. The full set of models will be made available electronically.

We summarize our results in Table 3 with determinations of the mass, radius, age, size of convective envelope, and the eddy turnover timescale at the base of the convective envelope for each star (See §4.1). The error bars indicate the range of values obtained in models with $\frac{\delta Y}{\delta Z} \in[1.5,3.5]$ and each of the input parameters, $L_{*}, T_{\text {eff }}$, and $[\mathrm{Fe} / \mathrm{H}]$ varied within the ranges specified below for each star. These error bars do not include the uncertainty in the choice of physical models discussed in $\S 2.1$. For our best model of each star we also list the dimensionless gyration radius of the star, the dimensionless gyration radius of the convective envelope (See §4.1) and the mass of the convective envelope at the ZAMS.

\subsection{Pegasi}

The planet around 51 Pegasi was discovered by Mayor \& Queloz (1995) and has since been confirmed by both Marcy et al. (1997) and Horner et al. (1997). The SFSU team has the cleanest data, giving an rms scatter of $5.2 \mathrm{~m} \mathrm{~s}^{-1}$ about a Keplerian fit with a semi-amplitude of $55.9 \pm 0.8 \mathrm{~m} \mathrm{~s}^{-1}$. They calculate an orbital period of $4.2311 \pm 0.0005 \mathrm{~d}$ and an eccentricity of $0.012 \pm 0.010$. The possibility of a second companion has been carefully examined. The SFSU planet search should already be able to detect planets with $m \sin i \simeq 1 M_{J}$ within $2 \mathrm{AU}$. A longer temporal baseline of observations is necessary to extend this limit (Marcy et al. 1997). Several teams have searched for periodic spectral line bisector variations. Although there is no convincing evidence (Horner et al. 1997; Hatzes, Cochran, \& Cohns-Krull 1997; Gray 1998; Hatzes, Cochran, \& Bakker 1998), the possibility of exciting non-radial stellar pulsations in 51 Peg-like systems remains interesting (Willems et al. 1997; Terquem et al. 1998), and these may become detectable in the future.

We calculated our models with the values from Gonzalez (1998a): $T_{\text {eff }}=5750 \pm 75 \mathrm{~K}$ and $[\mathrm{Fe} / \mathrm{H}]=0.21 \pm 0.06$. The consistent determinations of these parameters from many observations (See Table 3 ) is comforting. We obtain a best model of $51 \mathrm{Peg}$ with a mass of $1.05_{-0.08}^{+0.09} M_{\odot}$, radius of $1.16 \pm 0.07 R_{\odot}$, and an age of $7.6_{-5.1}^{+4.0}$ Gyr. We find that $51 \mathrm{Peg}$ has a convective envelope $\left(M_{c e} \simeq 0.023_{-0.006}^{+0.007} M_{\odot}\right)$ only slightly larger than that of the sun $\left(M_{c e} \simeq 0.0174 M_{\odot}\right)$. The eddy turnover timescale calculated as in Rasio et al. (1996) is $\tau_{c e} \simeq 18.6 \pm 2.5 \mathrm{~d}$ which is close to the expected value for main-sequence solar-type stars, $\tau_{c e} \sim 20 \mathrm{~d}$. In addition, we can follow the history of the convective envelope. We find that the mass of convective envelope on the ZAMS was $M_{\mathrm{ce}, \mathrm{ZAMS}} \simeq 0.037 M_{\odot}$.

As a consistency check, we compare our predicted value of $\log g(\operatorname{cgs})=4.33 \pm 0.09$ with those determined observationally. We find general agreement, especially when we consider that several of the 
lower observed values have already been criticized in the literature. Fuhrmann, Pfeiffer, \& Bernkopf (1997) suggest that the low value of the Gratton, Carrenton, \& Castelli (1996) study is a consequence of their low $T_{\text {eff }}$, and that the McWilliam (1990) result should be discounted, as it depended on an earlier misclassification of $51 \mathrm{Peg}$ as a subgiant. Indeed, the reclassification of $51 \mathrm{Peg}$ as a dwarf has been verified by multiple observations. Fuhrmann et al. (1997) also discount the Xu (1991) value, as it is based on low resolution spectra. Finally, the Edvardsson et al. (1993a,b) result is superseded by their own more recent observations which appear in Tomkin et al. (1997). The remaining determinations are all consistent with our models.

The activity-age relation predicts an age of $10 \mathrm{Gyr}$ consistent with our result for $51 \mathrm{Peg}$ (Baliunas et al. 1997), and a rotation period of $29.6 \mathrm{~d}$, while a rotation period near $37 \mathrm{~d}$ has been observed (Baliunas et al. 1996). Combining a rotation period of $34 \pm 4 \mathrm{~d}$ with our radius, we compute an equatorial velocity, $v_{\mathrm{eq}}=1.78 \pm 0.23 \mathrm{~km} \mathrm{~s}^{-1}$. This can be combined with observed values of $v \sin i$ to provide estimates of the inclination and hence the actual companion mass. The determination of $v \sin i=1.4 \pm 0.3 \mathrm{~km} \mathrm{~s}^{-1}$ by Gonzalez (1998) is quite consistent with our calculated $v_{\text {eq }}$, implying $\sin i=0.8 \pm 0.2$ and $m \simeq 0.59 \pm 0.15 M_{\mathrm{J}}$. However, most recent determinations of $v \sin i$ are larger than the above computed $v_{\text {eq }}$ (See Table 4 ). It should be noted that both the values of Hatzes et al. (1997) and Francois et al. (1996) have already been corrected according to Gonzalez (1998). These measurements are either inconsistent or barely consistent with the radius and period, suggesting that $\sin i \sim 1$ and thus the companion mass is not very different from the minimum companion mass.

\section{2. $\tau$ Bootis}

The SFSU planet search discovered a planet around $\tau$ Boo in a $3.3128 \pm 0.0002 \mathrm{~d}$ near-circular orbit. There is an rms scatter of $13.9 \mathrm{~m} \mathrm{~s}^{-1}$ with occasional episodes of greater scatter about the Keplerian fit with semi-amplitude $469 \pm 5 \mathrm{~m} \mathrm{~s}^{-1}$. The scatter is significantly above the instrumental error, but cannot be explained by the presence of a second planet (Butler \& Marcy 1996). Several teams have searched $\tau$ Boo for periodic spectral line bisector variations, but none have been found (See §3.1).

We started with models based on the values of $T_{\text {eff }}$ and $[\mathrm{Fe} / \mathrm{H}]$ from the recent spectroscopic work of Gonzalez (1997a). In the process, we found that our models favored lower values of $T_{\text {eff }}$ and $[\mathrm{Fe} / \mathrm{H}]$, and so we considered other determinations of $T_{\text {eff }}$ and $[\mathrm{Fe} / \mathrm{H}]$. Upon reanalysis of his own data, Gonzalez has refined his estimate of $T_{\text {eff }}$ to $6550 \pm 100 \mathrm{~K}$ (Gonzalez 1997b). He claims that his data might be consistent with $T_{\text {eff }}=6400 \mathrm{~K}$, but that they are inconsistent with $T_{\text {eff }}=6300 \mathrm{~K}$. There may be a significant difference between spectroscopic and photometric determinations of $T_{\text {eff }}$ and $[\mathrm{Fe} / \mathrm{H}]$ (Gonzalez 1997a). The small number of metal-rich stars used in the calibration of photometric estimators is one possible explanation for the difference in spectroscopic and photometric estimates for $\tau$ Boo (Gonzalez 1997a). Alternatively, if $\tau$ Boo has a convective envelope, the tidal torque from a planet in a $3.3 \mathrm{~d}$ orbit could drive the envelope to rotate much more rapidly than normal, thereby driving activity on the stellar surface and altering the observed spectral characteristics of the star. However there is a more likely explanation: the high X-ray luminosity, radial velocity noise, young age, and observed rotation period are all consistent will $\tau$ Boo being a young star that is still rotating rapidly. A rotation period as short as $3.3 \mathrm{~d}$ can cause significant discrepancies between spectroscopic and photometric determinations of $T_{\text {eff }}$ and $[\mathrm{Fe} / \mathrm{H}]$. The rapid rotation will broaden spectral lines and slightly redden the color. This, like the high metallicity of $\tau$ Boo, can affect the continuum and thus all the line depths and widths. Photometric estimates for such rapidly rotating stars can also be adversely affected. 
We explored a range of $T_{\text {eff }}$ and $[\mathrm{Fe} / \mathrm{H}]$ significantly larger than the uncertainties in the Gonzalez (1997a,b) observations require. YREC was unable to construct any self-consistent models of $\tau$ Boo in the range of 1.0 to $1.8 M_{\odot}$ using $T_{\text {eff }} \geq 6600 \mathrm{~K}$ and $[\mathrm{Fe} / \mathrm{H}] \gtrsim 0.13$, the spectroscopic value from Gonzalez (1997a). If we adopt $T_{\text {eff }}=6400 \pm 100$ and $[\mathrm{Fe} / \mathrm{H}]=0.25 \pm 0.09$, then we arrive at a mass of $1.37 \pm 0.08 M_{\odot}$, a radius of $1.41_{-0.09}^{+0.10} R_{\odot}$ and an age of $1.2_{-0.8}^{+1.2}$ Gyr. Our models predict $\log g=4.27_{-0.07}^{+0.05}$, which is in agreement with most of the observations, but slightly inconsistent with the Gonzalez (1997a) value of $4.5 \pm 0.15$. The most interesting result from our models of $\tau$ Boo is the likely absence of a convective envelope. We find thin convective envelopes only in models with $T_{\text {eff }} \lesssim 6350 \mathrm{~K}$ or $[\mathrm{Fe} / \mathrm{H}] \lesssim 0.07$ (See Table $7)$.

The activity-age relation predicts an age of $\sim 2$ Gyr (Baliunas et al. 1997), consistent with our age. The Mount Wilson HK Project has revealed that $\tau$ Boo has chromospheric emission periods of approximately $3.5 \pm 0.5 \mathrm{~d}, 117 \mathrm{~d}$, and $11.6 \mathrm{yr}$ (Baliunas et al. 1997). The shortest period is believed to be the rotation period, which corresponds very nearly to the orbital period of the planet. That has led Marcy et al. (1997) and Drake et al. (1998) to suggest the possibility that the star may have been tidally spun up. If we assume the star's rotation period is synchronized with the planet's orbital period and combine the $3.3 \mathrm{~d}$ rotation period with our determinations of radius, then we calculate $v_{\text {eq }}=21.7_{-1.4}^{+1.1} \mathrm{~km} \mathrm{~s}^{-1}$. This is consistent with all determinations of $v \sin i$ and suggests that $\sin i \sim 0.67_{-0.06}^{+0.07}$, implying $m=7.1 \pm 0.8 M_{\mathrm{J}}$.

\section{3. $v$ Andromedae}

The SFSU team detected a companion to $v$ And in a $4.611 \pm 0.005 \mathrm{~d}$ near-circular orbit. There is a $12.1 \mathrm{~m} \mathrm{~s}^{-1}$ rms scatter about the Keplerian fit of semi-amplitude $74.1 \pm 0.4 \mathrm{~m} \mathrm{~s}^{-1}$. This scatter is well above the instrumental error and could be either intrinsic to the star or indicative of a second planet. Early observations reported a significant eccentricity of $0.109 \pm 0.040$ (Butler \& Marcy 1996), but the long-term trend in the residuals complicates the measurement of the eccentricity. Thus, the current uncertainty is likely larger than the quoted error bar (Marcy 1998).

Based on the observations of Gonzalez (1997a), we calculate a mass of $1.34_{-0.12}^{+0.07} M_{\odot}$, radius of $1.56_{-0.10}^{+0.11} R_{\odot}$, and age of $2.6_{-1.0}^{+2.1} \mathrm{Gyr}$. The convective envelope is very shallow with $M_{c e} \simeq 0.002_{-0.002}^{+0.003} M_{\odot}$ and the eddy turnover time is correspondingly short, $\tau_{c e} \simeq 6.8_{-6.8}^{+2.3} \mathrm{~d}$.

The activity-age relation predicts an age of 5 Gyr, consistent with our results (Baliunas et al. 1997). Based on Ca II emission, $v$ And is expected to have a rotation period of $\sim 12 \mathrm{~d}$ (Baliunas et al. 1997). If accurate, one can infer an equatorial velocity $v_{\text {eq }} \sim 6.6 \mathrm{~km} \mathrm{~s}^{-1}$. This value is low compared to observed values of $v \sin i \sim 9 \mathrm{~km} \mathrm{~s}^{-1}$ (See Table 8). This suggests a significant error in either the observed value of $v \sin i$ or the estimated rotation period. If we were to take the reported $v \sin i$ at face value, then we would expect a rotation period $\lesssim 6 \mathrm{~d}$.

Our models give $\log g=4.18_{-.10}^{+.07}$, which is consistent with all but one of the observed values of $\log g$. Blackwell et al. (1990) have used the infrared flux method to calculate an angular diameter of $1.103 \pm 0.044$ mas, which, combined with the Hipparcos parallax, gives $R_{*}=1.60 \pm 0.08 R_{\odot}$, in agreement with our models. This corrects some previous estimates based on the Blackwell et al. (1990) data and older parallax data (most notably 56.8 \pm 4.1 mas from van Altena et al. 1995 which led to incorrect estimates of $T_{\text {eff }}$ and the radius). 


\section{4. $\rho^{1}$ Cancri}

The SFSU team has discovered a companion around $\rho^{1}$ Cnc in a near-circular orbit of period $14.648 \pm 0.0009 \mathrm{~d}$. They find a Keplerian fit to the radial velocity with a semi-amplitude of $77.1 \pm 0.9 \mathrm{~m}$ $\mathrm{s}^{-1}$ and a rms scatter of $12.0 \mathrm{~m} \mathrm{~s}^{-1}$. In addition, there appears to be a long-term trend in the residuals, possibly indicative of a second planet with mass $\sim 10 M_{\mathrm{J}}$ and a period of $\sim 20 \mathrm{yr}$ (Marcy \& Butler 1998). Previously, McAlister et al. (1993) had searched for a luminous companion in a similar orbit using speckle observations, but found none.

Initially, we attempted to construct models based on the stellar parameters measured by Gonzalez (1998ab). However, we found that this value of the temperature, $T_{\text {eff }}=5150 \pm 75 \mathrm{~K}$, is too low to match any model of $\rho^{1}$ Cnc, even when evolved for 20 Gyr.

There are several possible explanations for the apparent inconsistency of the various available data for $\rho^{1}$ Cnc. One possibility is that $\rho^{1} \mathrm{Cnc}$ is actually a subgiant. Indeed, $\rho^{1}$ Cnc's spectra closely matches that of $\delta$ Eri, a subgiant with $[\mathrm{Fe} / \mathrm{H}] \simeq-0.15$ (Baliunas et al. 1997)]. Gonzalez (1998a) agrees that the spectrum is suggestive of a subgiant, as is the low surface gravity. However our models, as well as the isochrones used by Gonzalez (1998a), would predict an extremely large age, of $\gtrsim 12$ Gyr.

Gonzalez (1998) proposes another possible explanation: the $\rho^{1}$ Cnc system may be an unresolved stellar binary viewed nearly face-on, despite the a priori low probability of such a viewing angle. Gonzalez (1998) suggests monitoring the line profile variations to test this hypothesis. We discuss this possibility further in $\S 4.1 .2$, and find this hypothesis unattractive.

Another possible explanation is an error in the stellar models. The observed value of $[\mathrm{Fe} / \mathrm{H}]$, $+0.45 \pm 0.03$ for $\rho^{1} \mathrm{Cnc}$ is quite extreme and the use of $\delta Y / \delta Z=2.5 \pm 1.0$ to determine $\mathrm{Y}$ for our models (as well as the isochrones of Schaller et al. (1992) and Scaerer et al. (1993a,b) used by Gonzalez 1998) may not be appropriate for such high metallicity stars. While this constraint on $\delta Y / \delta Z$ is believed to be reasonable for most solar-like stars, the calibration is based on lower metallicities and this linear relationship may not be adequate for high values of $\mathrm{Z}$.

Finally, it is possible that the observations have larger errors than those quoted. In particular, a higher $T_{\text {eff }}$ would restore consistency. However, since the observed value of $[\mathrm{Fe} / \mathrm{H}]$ is correlated with $T_{\text {eff }}$, the observed metallicity of $\rho^{1}$ Cnc would then increase from its (already high) quoted value (Gonzalez 1996, 1998ab). If we adopt $T_{\text {eff }}=5300 \pm 75$ and $[\mathrm{Fe} / \mathrm{H}]=0.45 \pm 0.03$, then we find a mass of $0.95_{-0.09}^{+0.11} M_{\odot}$ and a radius of $0.93_{-0.03}^{+0.02} R_{\odot}$. The convective envelope $\left(M_{c e} \simeq 0.046_{-0.006}^{+0.004} M_{\odot}\right)$ is significantly larger than solar and the eddy turnover time is slightly longer, $\tau_{c} \simeq 26.7_{-2.2}^{+1.2} \mathrm{~d}$. The age is very sensitive to $Y$ and thus is not well constrained. This explanation of our difficulties in modeling $\rho^{1} \mathrm{Cnc}$ is supported by the more recent observations of $\rho^{1}$ Cnc by Fuhrmann et al. (1998) and Gonzalez (1998b). Given these recent observations and the difficulties with the alternative explanations, we find this explanation the most attractive. Although the Fuhrmann et al. (1998) and Gonzalez (1998b) observations consistently support a larger $T_{\text {eff }}$, the unknown helium fraction still prevent us from obtaining an accurate determination of the age. Fuhrmann et al. (1998) obtained a maximum age based on their own observations. In addition to their observations having the largest $T_{\text {eff }}$, they have assumed a solar helium abundance. If we use their observational data and assume a solar helium abundance, then we obtain a similar upper limit on the age. However, if we use their observational data, but assume $\delta Y / \delta Z=2.5 \pm 1.0$, then we can no longer impose such a constraint. Thus,

${ }^{2}$ Cayrel de Strobel et al. 1997 lists many values of $[\mathrm{Fe} / \mathrm{H}]$ for $\delta$ Eri, ranging from -0.27 to 0.33 . 
their determination of a maximum age for $\rho^{1}$ Cnc is only valid if $\rho^{1}$ Cnc has a near solar helium abundance.

In light of the problems modeling $\rho^{1}$ Cnc, we consider several other pieces of observational data. Our models give $\log g=4.50_{-0.07}^{+0.04}$, significantly higher than the Gonzalez $(1996,1998)$ value of $4.15 \pm 0.05$, but in agreement with other observations (See Table 10). This is expected since $\log g$ is correlated with $T_{\text {eff }}$. The activity-age relation suggests an age of $\sim 5$ Gyr (Baliunas et al. 1997). The observed rotation period of $41.7 \mathrm{~d}$ (Baliunas et al. 1997) can be combined with our radius to obtain $v_{\text {eq }}=1.12 \pm 0.03 \mathrm{~km} \mathrm{~s}^{-1}$, barely consistent with the observed value of $v \sin i, 1.4 \pm 0.5 \mathrm{~km} \mathrm{~s}^{-1}$. The Barnes and Evans relationship (Barnes et al. 1978) gives an angular diameter of $0.790 \pm 0.032$ mas, which we combine with the Hipparcos parallax to calculate a radius of $1.06 \pm 0.04 R_{\odot}$, larger than the radii we find in our models.

\section{5. $\rho$ Coronae Borealis}

The Advanced Fiber Optic Echelle (AFOE) spectrograph team discovered a companion orbiting $\rho \mathrm{CrB}$ in a $39.645 \pm 0.088 \mathrm{~d}$ near-circular orbit. They fit a Keplerian curve of semi-amplitude $67.4 \pm 2.2 \mathrm{~m} \mathrm{~s}^{-1}$ to their data leaving a rms scatter of $9.2 \mathrm{~m} \mathrm{~s}^{-1}$ (Noyes et al. 1997). More recent data indicate that the orbit may have an eccentricity of $0.15 \pm 0.03$ (Marcy 1998).

We find the metallicity $[\mathrm{Fe} / \mathrm{H}]=-0.29 \pm 0.05$ quoted by Gonzalez (1998a) to be only marginally consistent with our models. We find consistent models only at the upper end of the quoted error bar. Using the observations of Gonzalez (1998a), but with a slightly higher $[\mathrm{Fe} / \mathrm{H}]=-0.23 \pm 0.06$, we calculate a mass of $0.89_{-0.04}^{+0.05} M_{\odot}$, radius of $1.35_{-0.08}^{+0.09} R_{\odot}$, and age of $14.1_{-2.4}^{+2.0}$ Gyr. We calculate $M_{c e} \simeq 0.033_{-0.009}^{+0.011}$ and $\tau_{c} \simeq 21.5_{-2.8}^{+2.9}$. We find general agreement between our computed $\log g=4.13_{-0.06}^{+0.07}$ and the observed values (See Table 12).

The activity-age relation predicts an age of 6 Gyr (Noyes et al. 1997), significantly younger than in our models. While our old age is worrisome, it is not extremely sensitive to any of the input parameters. For example, insisting that the age is $\lesssim 10 \mathrm{Gyr}$, would require a $2-\sigma$ error in both the temperature and the metallicity. No rotation has been observed, but based on the Ca II flux, the rotation period is predicted to be $\sim 20 \mathrm{~d}$ (Noyes et al. 1997). Combining this period and our radius, we compute $v_{\text {eq }} \sim 3.4 \mathrm{~km} \mathrm{~s}^{-1}$. This is consistent with the observed values of $v \sin i \sim 1.5 \mathrm{~km} \mathrm{~s}^{-1}$, but an accurate estimate of $\sin i$ cannot be obtained given the large uncertainty in the rotation period.

\section{Implications for Planet Formation and Evolution}

\subsection{Tidal Dissipation}

Our models do not change the main conclusions of Rasio et al. (1996) concerning the importance of orbital decay driven by tidal dissipation in the $51 \mathrm{Peg}$ system, but they do alter some of the quantitative estimates (See Fig. 1). The planets around $\tau$ Boo and $v$ And have longer timescales for orbital decay, since, as our models show, these stars have at most a very shallow convective envelope. The larger semi-major axes of the planets around $\rho^{1} \mathrm{Cnc}$ and $\rho \mathrm{CrB}$ increase their timescale for orbital decay. Thus, our models show that all the known extrasolar planets have stable orbits, in the sense that the orbital decay timescale is long compared to the main-sequence lifetime of their star.

Since orbital circularization is dominated by tidal dissipation in the planet, the only relevant stellar 
parameter for circularization is the stellar mass, as it affects our determination of the mass of the planet and the semi-major axis from observations. Parameters for the planet, such as the radius and the tidal dissipation factor, $Q$, are less certain, but we can estimate them using Jupiter as a guide (Rasio et al. 1996; Lubow, Livio, \& Tout 1997). Tidal dissipation in the planet could circularize an eccentric orbit for the 51 Peg, $\tau$ Boo, and $v$ And systems, and perhaps the $\rho^{1}$ Cnc system as well. Tidal dissipation in the planet could not have circularized an eccentric orbit in the case of $\rho \mathrm{CrB}$.

Tidal dissipation in the star also tends to spin up the star towards synchronization with the orbital motion of the planet. since radial velocity surveys can only determine the $v \sin i$, some of the detected systems may actually contain low-mass stellar companions in orbits that happen to be viewed nearly face-on $(\sin i \ll 1)$. However, the probability of finding a $1 M_{\odot}$ companion with $m \sin i$ as low as $1 M_{\mathrm{J}}$ or $10 M_{\mathrm{J}}$ is only one in $\sim 2 \cdot 10^{6}$ or $\sim 2 \cdot 10^{4}$, respectively. Since the SFSU planet search has already found 6 systems by monitoring a target list of only 120 stars, the spectroscopic binary explanation is extremely unlikely, but cannot be strictly ruled out. In principle, astrometry could be used to constrain the maximum companion mass. However, this is extremely difficult for short-period systems, since the position wobble is so small. For example, assuming a low mass companion to $51 \mathrm{Peg}$, the amplitude of the induced wobble is only $3 \cdot 10^{-3} / \sin i$ mas. Sophisticated space-based interferometers will be necessary to measure such small effects. Absence of X-ray activity can also be used to confirm that a companion is substellar, since close spectroscopic binaries are usually observed to have significant X-ray luminosities. However, a young stellar age or rapid rotation also correlate with strength of X-ray emission (see e.g., Pravdo et al. 1996).

Here we focus on the tidal constraints, which can place an upper limit on the mass of companions in short-period systems. For the planet to survive, the orbital decay timescale must be large compared to the age of the system. If the companion has not yet spun up the stellar rotation period to match its orbital period, then another constraint can be imposed. The maximum companion mass, as determined from the timescales for either orbital decay or spin-up, can be calculated from the stellar parameters. Following standard tidal dissipation theory (e.g., Zahn 1977; Zahn \& Bouchet 1989; Rasio et al. 1996), we calculate the timescale for a planet to spin up the whole star

$$
\tau_{\mathrm{su}}^{*}=\frac{k_{*}^{2} M_{*} R_{*}^{2}}{m a^{2}} \tau_{\mathrm{a}},
$$

where $k_{*}=\left(I_{*} / M_{*} R_{*}^{2}\right)^{1 / 2}$ is the dimensionless gyration radius, $M_{*}$ is the mass of the star, $R_{*}$ is the stellar radius, $m$ is the mass of the planet, $a$ is the semi-major axis, and $\tau_{\mathrm{a}}$ is the timescale for orbital decay,

$$
\tau_{\mathrm{a}}^{-1}=\frac{f}{\tau_{c}} \frac{M_{\mathrm{ce}}}{M_{*}} q(1+q)\left(\frac{R_{*}}{a}\right)^{8} .
$$

Here $q=m / M_{*}$ is the mass ratio, $f$ is a numerical factor of order unity, and $\tau_{\mathrm{c}}$ is the eddy turnover timescale. Following Rasio et al. 1996, we estimate $\tau_{\mathrm{c}}$ by

$$
\tau_{\mathrm{c}}=\left[\frac{M_{\mathrm{ce}} R_{\mathrm{ce}}\left(R_{*}-R_{\mathrm{ce}}\right)}{3 L_{*}}\right]^{1 / 3}
$$

where $L_{*}$ is the stellar luminosity, $M_{\text {ce }}$ is the mass of the convective envelope, and $R_{\text {ce }}$ is the radius at the base of the convective envelope. When the tidal pumping period (half the orbital period) is small compared

\footnotetext{
${ }^{3}$ Note the obvious typographical error in Eq. 2 of Rasio et al. (1996). Here we use standard tidal dissipation theory based on the weak friction approximation with eddy viscosity as the dominant dissipation mechanism in stars with convective envelopes (Zahn 1977; Rasio et al. 1996). Although the theory remains controversial (see, e.g., Goodman \& Dickson 1998; Tassoul \& Tassoul 1997), there is empirical support for some of its main predictions (Verbunt \& Phinney 1995; Zahn 1992).
} 
to the eddy turnover time in the convective envelope, we expect that the efficiency of the tidal dissipation will be reduced. Thus, for fast tidal pumping, we reduce the factor $f$ according to

$$
f=f^{\prime} \min \left[1,\left(\frac{P}{2 \tau_{\mathrm{c}}}\right)^{2}\right] .
$$

Since the exact form of this correction is rather uncertain (see Goodman \& Oh 1997 and references therein), we compute tidal decay and spin-up timescales using both $f=1$ and $f$ as given by Eq. 4 with $f^{\prime}=1$.

An even stronger constraint can be imposed if the spin up of the convective envelope is assumed to occur independently from the interior. In the limit of a thin convective shell, the spin-up timescale becomes

$$
\tau_{\mathrm{su}}^{\mathrm{ce}} \simeq \frac{M_{c e} R_{*}^{2}}{m a^{2}} \tau_{\mathrm{a}} \simeq \frac{k_{\mathrm{ce}}^{2}}{k_{*}^{2}} \tau_{\mathrm{su}}^{*} .
$$

In reality, the true spin-up timescale is likely to be somewhere between $\tau_{\mathrm{su}}^{\mathrm{ce}}$ and $\tau_{\mathrm{su}}^{*}$.

We now consider the importance of tidal effects in each system individually.

51 Peg. It has been suggested that the 51 Peg system could contain a stellar-mass companion, but that the system is being viewed nearly face-on (Kubat, Holmgren, \& Rentzsch-Holm 1998; Imbert \& Prevot 1998). Astrometric measurements with Hipparcos impose an upper limit of $\sim 500 M_{\mathrm{J}} \sim 0.5 M_{\odot}$ (Perryman et al. 1997). Diffraction limited ( $\sim 0.05$ arcsec) infrared speckle imaging with the Keck telescope searched mainly for $\mathrm{M}$ dwarf companions and put limits on any such companions. These detection limits give a range of maximum orbital separations from 0.75 AU for M3 dwarfs to 23 AU for M7 dwarfs (Marcy et al. 1997). The Palomar Testbed Interferometer has also searched the 51 Peg system and determined a maximum companion mass of $0.22 M_{\odot}$ (Boden et al. 1998). Low levels of X-ray emission also indicate that the 51 Peg system is unlikely have a stellar-mass companion (Pravdo et al. 1996).

We can put another constraint on the maximum companion mass based on the slow rotation rate of the star implying $\tau_{\mathrm{su}} \gg \tau_{\mathrm{MS}}$. We calculate $\tau_{\mathrm{su}}^{*} \sin ^{2} i \simeq 8.4 \cdot 10^{13} \mathrm{yr}$. Comparing this to the best model age of $51 \mathrm{Peg}$, we get a maximum companion mass of $46 M_{J}$. If we set $f=1$, ignoring the reduction in efficiency from the rapid pumping period, then we obtain $\tau_{\mathrm{su}}^{*^{\prime}} \sin ^{2} i \simeq 1.1 \cdot 10^{12} \mathrm{yr}$, which yields a maximum mass of $5.5 M_{\mathrm{J}}$. We also calculate $\tau_{\mathrm{su}}^{\mathrm{ce}} \sin ^{2} i \simeq 1.2 \cdot 10^{13} \mathrm{yr}$ and $\tau_{\mathrm{su}}^{\mathrm{ce}} \sin ^{2} i \simeq 1.6 \cdot 10^{11} \mathrm{yr}$, with corresponding maximum companion masses of $19 M_{\mathrm{J}}$ and $2.1 M_{\mathrm{J}}$, respectively (See Fig. 2).

$\tau$ Boo. The most interesting result from our models of $\tau$ Boo is the likely absence of a convective envelope. We find convective envelopes only in models with $T_{\text {eff }} \lesssim 6350 \mathrm{~K}$ or $[\mathrm{Fe} / \mathrm{H}] \lesssim 0.07$. Even in these models, the convective envelope is extremely thin. The spectral line bisectors seen by Hatzes et al. (1997) and others display a slight curvature, consistent with a very thin convective layer, which our models might not capture and which would not be significant for tidal dissipation. Even if we include our models with the most significant convective envelopes $\left(T_{\mathrm{eff}}=6200 \mathrm{~K},[\mathrm{Fe} / \mathrm{H}]=0.16\right)$, we can place an upper limit on the size of the convective zone of $M_{c e} \lesssim 0.003 M_{\odot}$ (See Table 3).

While this result does not affect the possibility of tidal dissipation in the planet circularizing the orbit as discussed in Rasio et al. (1996) and Rasio \& Ford (1996), it does have significant implications for the spin history of $\tau$ Boo. Marcy et al. (1997) and Drake et al. (1998) suggest that the planet is likely to have tidally spun up the star. Our models indicate that $\tau$ Boo does not have a convective envelope and thus would not have been spun up by a close companion. In fact, a rotation period of $3.3 \mathrm{~d}$ is entirely consistent the observed normal rotation rates of young F7 stars with fully radiative envelopes (Gray \& Nagor 1985). 
Thus, the approximate equality of the observed rotation period and the orbital period is most likely a coincidence and not the result of tidal spin-up.

If we insist that tidal dissipation synchronized the stellar rotation period, then it must have occurred much earlier in $\tau$ Boo's life, when the star had a large convective envelope. Our models indicate that $\tau$ Boo's convective envelope starts to shrink rapidly near $\sim 2 \cdot 10^{6} \mathrm{yr}$ and is reduced to $\lesssim 4 \cdot 10^{-3} M_{\odot}$ after $\sim 1.5 \cdot 10^{7} \mathrm{yr}$. For a short time, $\sim 2-3 \cdot 10^{7} \mathrm{yr}$, the synchronization timescale is $\sim 2 \cdot 10^{6} \mathrm{yr}$, providing a window of opportunity for tidal synchronization. By $\sim 3 \cdot 10^{7} \mathrm{yr}$, the convective envelope has shrunk to less than $10^{-4} M_{\odot}$. Thus, tidal synchronization would require that the planet be already formed and in a short-period orbit before this time. Indeed, it has been suggested that the planet may have migrated to its present orbit and stopped at an orbital period synchronized with the stellar rotation, because of the tidal force exerted on the orbit (Lin et al. 1996; Trilling et al. 1998). Since the system is quite young, it is possible that the star has not had enough time to spin down significantly after the disappearance of the convective envelope.

$v$ And. There is no measured rotation period for $v$ And. Although most of our models of $v$ And do have a small convective envelope, some of the models we constructed for $T_{\text {eff }}=6350 \mathrm{~K}$ do not. If we adopt our best model, we find that the constraint $\tau_{\mathrm{su}}^{*}<\tau_{\mathrm{MS}}$ would impose a limit of $43 M_{\mathrm{J}}$ on the companion mass, while ignoring the reduction in efficiency from the rapid pumping period would impose a limit of $15 M_{\mathrm{J}}$. The constraint that $\tau_{\mathrm{su}}^{\mathrm{ce}}<\tau_{\mathrm{MS}}$ would impose limits of $5.5 M_{\mathrm{J}}$ for $f^{\prime}=1$ and $1.9 M_{\mathrm{J}}$ for $f=1$.

The latest radial velocity data indicate that the planet around $v$ And may have a significantly eccentric orbit, $e \simeq 0.1$ (Marcy 1998). If this eccentricity is confirmed, then the non-circular orbit, combined with the survival of the planet, could place constraints on the planet's structure. Following Rasio et al. (1996) we calculate the timescales for synchronization of the planet's rotation and for orbital circularization. Assuming that the planet is identical to Jupiter, we find that the rotation of the planet should be synchronized with the orbit and that the circularization timescales is comparable to the age of the system. Since both these timescale are very sensitive to the radius of the planet, we can turn the observed non-zero eccentricity into an upper limit on the planet's radius. Taking the age to be 2.6 Gyr and still assuming the same dissipation factor, $Q$, as Jupiter, we find $R_{\mathrm{p}} \lesssim 1.4 R_{J}$. Unfortunately, the uncertainty in the age of the system, and even more in $Q$, prevents this from being a stringent constraint at this time.

$\rho^{1}$ Cnc: We were not able to construct any models of $\rho^{1}$ Cnc with a temperature as low as that observed by Gonzalez (1998ab). One possible explanation is that the $\rho^{1}$ Cnc system might be an unresolved stellar binary viewed nearly face-on (Gonzalez 1998ab).

Since the star has an observed rotation period of $42 \mathrm{~d}$, which is longer than the orbital period, we can calculate the maximum companion mass which would not have synchronized the stellar rotation period. We calculate $\tau_{s u}^{*} \sin ^{2} i \simeq 1.4 \cdot 10^{15} \mathrm{yr}$. Comparing this to the age of $\rho^{1} \mathrm{Cnc}$, we get a maximum companion mass of $\sim 0.33 M_{\odot}$. If we set $f=1$, ignoring the reduction in efficiency from the rapid pumping period, then we obtain $\tau_{\mathrm{su}}^{*^{\prime}} \sin ^{2} i \simeq 1.1 \cdot 10^{14} \mathrm{yr}$, which yields a maximum mass of $91 M_{\mathrm{J}}$. We also calculate $\tau_{\mathrm{su}}^{\mathrm{ce}} \sin ^{2} i \simeq 3.5 \cdot 10^{14} \mathrm{yr}$ and $\tau_{\mathrm{su}}^{\mathrm{ce}} \sin ^{2} i \simeq 2.5 \cdot 10^{13} \mathrm{yr}$, which give maximum companion masses of $\sim 0.17 M_{\odot}$ and $46 M_{\mathrm{J}}$, respectively (See Fig. 4). While this excludes the possibility of most stellar mass companions, we can not completely exclude the possibility of a nearly face-on stellar companion of mass $\lesssim 0.3 M_{\odot}$ (Gonzalez 1998ab). Additionally, since the companion is very close to $\rho^{1} \mathrm{Cnc}$, it may be contaminating the spectra and distorting the observed parameters sufficiently to affect the maximum mass we calculated here.

$\rho$ CrB. While tidal dissipation in the planets could circularize the orbits in $51 \mathrm{Peg}, \tau$ Boo, $v$ And, and possibly also $\rho^{1} \mathrm{Cnc}$, the planet around $\rho \mathrm{CrB}$ has too large a semi-major axis for tidal dissipation to 
be significant anywhere. Indeed, the orbit is observed to have an eccentricity of $0.15 \pm 0.03$ (Marcy 1998). Depending on the orbital parameters of the secondary star, secular perturbations may be able to produce the observed eccentricity in the orbit of the lower mass companion. Alternatively this eccentricity may have been produced by a dynamical interaction with another planet. Alternatively, an interaction with a circumstellar disk could induce an eccentricity, but only if the planet is significantly more massive than its minimum mass (Artymowicz 1992).

\subsection{Metallicity Enhancements}

\subsubsection{Observational Evidence}

Observed metallicities are listed in Table 2. Of the five short period planets, all but $\rho$ CrB have $[\mathrm{Fe} / \mathrm{H}]>0.15$. These five stars have a mean metallicity of +0.18 . If we consider the four stars with planets in the shortest period orbits, then the average metallicity increases to +0.29 . Additionally, six of the seven stars known to have companions with $m \sin i \lesssim 5 M_{\mathrm{J}}$ are metal rich relative to the sun and they have a mean metallicity of +0.14 . These observations have led to speculations that there may be a relationship between stars with higher metallicities and stars with planets.

When analyzing these metallicities, one must be careful to consider the survey population from which the stars were selected. As an example, consider the SFSU planet search, which has monitored 120 stars. Of those, 67 stars are also in the Cayrel de Strobel et al. (1997) catalogue. Looking at the intersection of these two lists, we find that only $8 \%$ have an average $[\mathrm{Fe} / \mathrm{H}]>0.15$. Since all the stars known to harbor planets are $\mathrm{F}$ and $\mathrm{G}$ stars and it is more difficult to obtain accurate metallicities for $\mathrm{K}$ and $\mathrm{M}$ stars, it may be better to restrict our attention to the $61 \mathrm{~F}$ and $\mathrm{G}$ stars in the SFSU planet search. Of those, 47 appear in the Cayrel de Strobel et al. (1997) catalogue, and only about $5 \%$ have an average $[\mathrm{Fe} / \mathrm{H}]>0.15$. Both populations have a mean $[\mathrm{Fe} / \mathrm{H}]=-0.09$ with a standard deviation of 0.22 . Additional evidence for the a relationship between high metallicities and extrasolar planets comes from a comparison with the recent, nearly volume-limited metallicity survey of nearby solar-type stars by Favata et al. (1997). They find a mean $[\mathrm{Fe} / \mathrm{H}]$ of $-0.07 \pm 0.26$ for their full sample and $-0.12 \pm 0.27$ for the portion of the sample with $T_{\text {eff }}>5000 \mathrm{~K}$. After they attempt to correct for various biases, they find a peak at $[\mathrm{Fe} / \mathrm{H}] \simeq-0.23$ (Favata, Micela, \& Sciortino 1997). Extrasolar planets in wide binary systems for which the metallicity of each star can be accurately measured could provide useful information about the relationship between metallicity enhancements and planet formation. In the cases of $\tau$ Boo, $\rho^{1} \mathrm{Cnc}, v$ And, and $\rho$ CrB accurate determinations of the metallicity of the binary companion are not available. In the 16 Cygni system, spectroscopic analyses have revealed very similar metallicities for the two stars, but slight differences in the lithium and beryllium abundances (Gonzalez 1998; Lopez \& Taoro 1998). Since both these elements are involved in nuclear reactions, complications such as rotation can significantly affect the observed abundances. Thus, we cannot conclude that there is a significant difference without more careful study. As more extrasolar planets are discovered in wide binary systems, these comparisons may become very instructive.

Several factors make some stars better candidates for planet detections than others and introduce additional observational biases. For example, high chromospheric activity increases the scatter in the radial

\footnotetext{
${ }^{4}$ Cayrel de Strobel et al. (1997) catalogues observations of $[\mathrm{Fe} / \mathrm{H}]$ from the literature. When the catalogue lists multiple observations for a single star, we take the unweighted average.
} 
velocity measurements, decreasing the sensitivity of the planet search. Some planet searches intentionally exclude such stars, while others observe them in spite of the reduced detection efficiency. In either case, this introduces complicated selection effects. While the selection effects are not fully understood, it seems that high stellar metallicities may be correlated with the existence of planets, or at least with short period planets.

\subsubsection{Mechanisms to Accrete High-Z Material}

Several mechanisms that have been discussed in the context of extrasolar planet formation may lead to the accretion of high- $\mathrm{Z}$ material onto the parent star. While such theories might also explain the apparent correlation of short period planets with higher metallicities, the plausibility of such an explanation depends on many factors.

One factor is the type of material accreted onto the star. Accreting material from a gaseous disk will have little effect, since this material has essentially the same metallicity as material which formed the star. However, accreting a large number of terrestrial planets, asteroids, or planetesimals could produce a noticeable effect. Alternatively, accreting gaseous giant planets like Jupiter could also have a significant effect since these planets are thought to contain up to $\sim 10 M_{\oplus}$ of rocky material in their core.

The size of the stellar convective envelope at the time of accretion is also of critical importance, since the accreted material is rapidly diluted over the entire stellar convective region. In particular, if the accretion of high- $Z$ material occurred while the star was still on the PMS and had a large convective envelope, then there would be little effect on the stars' observed metallicities. To be efficient in increasing the surface metallicity, the accretion must take place sufficiently late in the stellar evolution when the outer convective envelope is shallow (See Fig. 5).

Finally, the particular type of accretion process which adds high-Z material to the star could also be important. If small amounts of high-Z material are gradually deposited from nearly circular orbits, then it might remain in the outer convective envelope. However, if a massive object entered the star from a highly eccentric orbit, it might penetrate more deeply into the star, thereby diluting the high-Z material over a larger mass fraction. Furthermore, if a rocky body is added to the star from a nearly circular orbit, it still might sink to a significant depth before being disrupted. Even if the high-Z material is not immediately mixed deep into the stellar interior, a layer of high-Z material would have a greater mean molecular weight, which could drive a thermal instability allowing it to penetrate deeper into the star. It is beyond the scope of this paper to take all of these process into account. Instead, we simply obtain an upper bound on the effects of adding high-Z material to the star by noting that any material added must have been diluted at least across the present convective zone.

Our models show that, at present, $51 \mathrm{Peg}, \rho^{1} \mathrm{Cnc}$, and $\rho \mathrm{CrB}$ all have significant convective envelopes, while $v$ And and $\tau$ Boo have either very thin convective envelopes or none at all (See Table 3 ). However, our best models of $v$ And and $\tau$ Boo indicate that they did have convective envelopes at the ZAMS (See

Table 3). Since the size of the current convective envelope imposes an upper limit on the effects of chemical pollution from accretion independent of when the accretion occurred, we will only consider $51 \mathrm{Peg}, \rho^{1} \mathrm{Cnc}$, and $\rho \mathrm{CrB}$. We discuss the effects of adding various high- $\mathrm{Z}$ materials to these stars, but with an original metallicity of $[\mathrm{Fe} / \mathrm{H}] \simeq 0$. This metallicity is already higher than most nearby solar-type stars, as discussed above. 
Gas Giants. Lin et al. (1996) proposed a model in which several multiple Jupiter-mass planets migrate inwards as a result of dissipation in a massive disk. Today, we are only able to observe the last of the planets which were fortunate enough to barely avoid spiraling into their parent star. We consider the effect of adding Jupiter-like giant planets with $10 M_{\oplus}$ cores of roughly chondritic composition (Anders \& Grevesse 1989). Starting with a solar metallicity star and the presently observed convective envelope, it would take $\sim 5$ and $\sim 15$ such Jupiter-like giant planets to raise the stellar metallicities to those observed in $51 \mathrm{Peg}$ and $\rho^{1} \mathrm{Cnc}$, respectively. However, in this model, the accretion takes place while the star still has a gaseous protostellar disk $\left(t \lesssim 10^{7} \mathrm{yr}\right)$. At this stage the star would still be on the PMS where it would have a much larger convective envelope. Therefore, much more mass would be necessary to raise their metallicities to those observed today (See Fig. 5).

In a similar model, a single massive giant planet $\left(\sim 4 M_{\mathrm{J}}\right)$ migrates towards the parent star and then undergoes Roche lobe overflow, stabilizing its orbit against spiraling further inward (Trilling et al. 1998). Again, gas giant material would be accreted onto the star, although this time the rocky core would not be accreted. Since Jupiter's atmosphere is only slightly enriched in heavy elements, this is not likely to alter a star's observed metallicity significantly. Additionally, if the migration is due to a viscous disk, the star will still be highly convective, rendering the accreted material inefficient in raising the surface metallicity.

Gaseous Disks: In both of the above models, a portion of the gas disk can also be accreted onto the star. The disk may have a metallicity slightly larger than the star, but this is not expected to significantly alter the observed stellar metallicity. Since both the disk mass and the disk metallicity will vary with time and neither are well known, a quantitative estimate for this mechanism would be difficult.

In contrast, adding rocky material (terrestrial planets, asteroids, or planetesimals) to the star is much more efficient at increasing the observed values of $[\mathrm{Fe} / \mathrm{H}]$, as they have a much higher metallicity than gaseous giant planets. Given the presently observed convective envelopes and an initially solar composition, it would require only $\sim 40 M_{\oplus}$ and $\sim 100 M_{\oplus}$ of chondritic material (Anders \& Grevesse 1989) to reach the observed metallicities of 51 Peg and $\rho^{1}$ Cnc, respectively. However, standard planet formation scenarios do not predict much more than $\sim 20 M_{\oplus}$ in terrestrial planets.

Planetesimals: Accretion of a large amount of solid material in the form of asteroids or planetesimals arises naturally in the model proposed by Murray et al. (1998). In this model giant planets can migrate inwards while scattering planetesimals into the parent star.

Since the early migration is rapid, the planet will not clear out the planetesimal disk until its migration slows. Thus we neglect any planetesimals that are scattered into the star until the planet reaches its final orbit. The mass accreted on the star is given by

$$
M_{\mathrm{acc}} \simeq f(a) \frac{m}{\alpha}
$$

where

$$
f(a) \simeq 0.15\left(\frac{a}{A U}\right)^{-0.374}
$$

is the fraction of planetesimals scattered onto the star by the planet at distance $a, m$ is the mass of the planet, and $\alpha$ is a parameter $\sim 0.5-1.0$, which must be weighted across multiple resonances (Murray et al. 1998; Hansen 1998). Note that $f(a)$ only accounts for planetesimals which become planet crossing before colliding with the star, i.e., planetesimals which collide with the star without ever becoming planet-crossing are neglected.

Using this prescription, we can estimate the mass of planetesimals which would be scattered into the 
star for $51 \mathrm{Peg}, \rho^{1} \mathrm{Cnc}$ and $\rho \mathrm{CrB}$. We find $0.4 M_{\mathrm{J}}\left(130 M_{\oplus}\right)$ for $51 \mathrm{Peg}$ and $0.6 M_{\mathrm{J}}\left(180 M_{\oplus}\right)$ for both $\rho^{1}$ $\mathrm{Cnc}$, and $\rho \mathrm{CrB}$. Since all this mass is in asteroids, this could lead to a significant and observable increase in the metallicity. Starting from a star with solar metallicity and the present convective envelope, adding $130 M_{\oplus}$ of asteroids to $51 \mathrm{Peg}$ or $180 M_{\oplus}$ of asteroids to $\rho^{1}$ Cnc increases the observed $[\mathrm{Fe} / \mathrm{H}]$ to 0.48 and 0.39 dex, respectively, assuming (our best model for the two stars). However, the requirement that the planetesimal disk still be present, implies that the stars are still young with convective envelopes larger than at present. The time it takes for a planet to migrate to a $0.05 \mathrm{AU}$ orbit in the Murray et al. (1998) scenario is $\lesssim 3 \cdot 10^{7} \mathrm{yr}$, but this depends on the model of the planetesimal disk. Once the planet stops migrating, the asteroids inside its orbit are quickly cleared out. As can be seen from Fig. 5, there is a window of opportunity at $2-3 \cdot 10^{7} \mathrm{yr}$ which is the right time to produce the observed metallicities. However, since the convective envelope disappears rapidly, this requires a very specific timing. Different models of the disk or the inclusion of additional resonances could destroy any careful tuning of parameters in a particular model adjusted to produce significant metallicities.

Terrestrial Planets: Rasio \& Ford (1996) and Weidenschilling \& Mazari (1996) proposed that 51 Peg-like planets could be produced by gravitational scattering off a second massive planet. If a significant number of planets are to wind up very close to their parent stars, a significant number should also have collided with their parent stars. However, it is unlikely that two planets in the same system would be scattered so close to their parent star. Thus, one would not expect to see increased metallicities in stars with 51 Peg-like planets due to the accretion of giant planets. Similarly, in the model of Kiseleva \& Eggleton (1997), secular perturbations of a binary companion induce large eccentricities which are later circularized. In either this model or the previous model, a giant planet acquires a very high eccentricity which would disrupt inner terrestrial planets, possibly making them collide with the parent star. These mechanisms have the advantage that they can deposit material onto the star after it has reached the main sequence and its convective envelope has diminished to near its present size. It would take a large amount of mass in terrestrial planets and asteroids for these mechanisms to increase the metallicity from solar to what is observed in $51 \mathrm{Peg}$ or $\rho^{1} \mathrm{Cnc}\left(\sim 40 M_{\oplus}\right.$ and $\sim 100 M_{\oplus}$, respectively). One would still expect the planet to scatter asteroids into the star, as in the Murray et al. (1998) scenario. However, here a particular disk mass or timing is not required.

\subsubsection{Polluted Stellar Models}

The possibility of a star having a surface metallicity significantly higher than its interior metallicity arises naturally in the context of planet formation. Thus, when constructing models based on observational data, we should realize that the chemical composition of the interior, which contains most of the stellar

mass, could deviate significantly from the composition observed on the stellar surface. For this reason, it is important to consider stellar models with interior metallicities somewhat lower than those observed.

We have constructed a few models to explore the effects of adding high-Z material to the surface of a star. We pick an initial metallicity for the entire star and evolve a stellar model slightly past the ZAMS. Then, we increase the metallicity in the outer convective envelope and continue to evolve the star on the main sequence.

First, we consider the effect of adding a Jupiter with $10 M_{\oplus}$ of high-Z material (in its rocky core, using the procedure described above) to the ZAMS Sun. We find that the effective temperature, luminosity, and radius increase by $0.55 \%, 2.6 \%$, and $0.19 \%$, respectively, while the mass of the convective envelope decreases 
by approximately $5.5 \%$. Similar models have been investigated in the context of the Solar neutrino problem and have been found to reduce the agreement between theoretical and observational determinations of solar p-mode frequencies (Jeffery, Mailey, \& Chambers 1997; Christensen-Dalsgaard \& Gough 1998; Bahcall 1989).

Next, we consider the consequences of a reduced interior metallicity for our models. Since we use observations of the luminosity, effective temperature, and surface metallicity, the radius will not be affected, but the mass, age, and size of the convective envelope will be. Generally, decreasing the interior metallicity from the observed surface metallicity causes the mass to decrease, but the age and the size of the convective envelope both increase. For example, if we take the observed parameters for $51 \mathrm{Peg}$, but set the interior metallicity to solar, then the we obtain in our new best model a mass of $0.94 M_{\odot}$, an age of $10.8 \mathrm{Gyr}$, and a convective envelope of mass $0.026 M_{\odot}$ (see Table 3 for comparison).

Finally, we have explored the possibility that a reduced interior metallicity could explain the difficulties encountered in constructing models that match the observed parameters for $\rho^{1}$ Cnc and $\tau$ Boo. In general, lowering the interior metallicity leads to a higher surface temperature in our models. Therefore, in the case of $\rho^{1} \mathrm{Cnc}$, this would make the discrepancy worse. However, this could bring our models of $\tau$ Boo closer to agreement with the observed effective temperature. If we demand that our models of $\tau$ Boo have $T_{\text {eff }}=6550 \mathrm{~K}$, as indicated by the recent observations of Gonzalez (1998a), then the difference between the interior and surface metallicities must be $\sim 0.35$ dex. Although possibly a coincidence, this is comparable to $\tau$ Boo's observed $[\mathrm{Fe} / \mathrm{H}]=0.34 \pm 0.09$. However, since we were able to construct models with a single metallicity for $T_{\text {eff }}=6500 \mathrm{~K}$, the uncertainty in the measurement of the effective temperature $(\simeq 100 \mathrm{~K})$ prevents us from placing a firm constraint on the interior metallicity. If we use the same parameters for $\tau$ Boo as discussed in $\S 3.2$, but with a solar metallicity interior, we find a mass of $1.29 M_{\odot}$, an age of 0.4 Gyr, and no significant outer convective envelope.

We find that a reduced interior metallicity could resolve the difficulty in constructing models that match the observed luminosity, temperature, and metallicity for $\tau$ Boo. In our calculations we have instantaneously distributed the accreted material across the convective envelope of the star and then evolved the star in this dual composition state. If the material is deposited on a dynamical timescale, then the star will temporarily be out of thermal equilibrium. A future calculation could evolve the star on a thermal timescale to follow the readjustment to a new thermal equilibrium. In addition, if the convective envelope has a higher metallicity than the interior, then the possibility of triggering a thermal instability that could drive high metallicity material into the stellar interior should be examined.

\section{Summary}

We have computed stellar models for nearby stars with close planetary-mass companions. Tables 5, $7,9,11$, and 13 show the properties of models constructed for different values of the observed parameters within measurement uncertainties. These models were used to calculate the stellar parameters and error bars summarized in Table 3. We will make a larger grid of stellar models available electronically to help interpret future stellar observations.

Using conventional tidal dissipation theory, we have studied tidal dissipation effects in these systems, based on the results of our models. We find that the orbital decay timescale is longer than the age of the star in all systems (See Figs. 1 and 3). The timescale for orbital circularization is shorter than the stellar ages in $51 \mathrm{Peg}, \tau$ Boo, and $v$ And, comparable to the stellar age in $\rho^{1} \mathrm{Cnc}$, but longer than the stellar 
age in $\rho \mathrm{CrB}$. We have also calculated maximum companion masses based on the lack of synchronization between the stellar rotation and the companion orbital period for $51 \mathrm{Peg}, v$ And and $\rho^{1}$ Cnc (See Figs. 2 and 4). For the most optimistic assumptions for the tidal coupling (in particular assuming that the tidal torque is exerted only on the outer convective envelope), we find $m \lesssim 2.1 M_{\mathrm{J}}, 1.9 M_{\mathrm{J}}$ and $46 M_{\mathrm{J}}$ for 51 Peg, $v$ And, and $\rho^{1}$ Cnc, respectively. For $\tau$ Boo the similarity between the stellar rotation period and the orbital period is likely a coincidence and not the result of tidal spin-up. If the significant eccentricity of the $v$ And system is confirmed, then we can place an upper limit on the radius of the planet, $R_{p} \lesssim 1.4 R_{\mathrm{Jup}}$.

We have examined the observational evidence for a correlation between the presence of a planet and a high stellar metallicity. We have evaluated the effects of accreting high-metallicity material onto a star in the context of various proposed mechanisms for producing short period systems. The accretion of gas giants or of material from a gaseous disk causes only minor metallicity enhancements if the stellar convective envelope is still large at the time of the accretion. Accretion of rocky material, such as planetesimals, asteroids, and terrestrial planets, could cause significant increases in observed metallicities if enough mass is available. An increased surface metallicity can also have a significant effect on stellar models and on the interpretation of observational data.

We are very grateful to Guillermo Gonzalez for several valuable discussions, reanalysis of his data, and for sharing papers in advance of publication. We thank Dimitar Sasselov for assistance in interpreting observational data. We also thank Brad Hansen, Matt Holman, and Norm Murray for their help in forming the estimates for their model in $§ 4.2$, and Sallie Baliunas, Robert Donahue, Scott Horner, and Geoff Marcy for useful comments. This work was supported by NSF Grant AST-9618116. E.B.F. is supported in part by the Orloff UROP Fund and the UROP program at MIT. F.A.R. is supported in part by an Alfred P. Sloan Research Fellowship. A.S. is supported in part by the Natural Sciences and Engineering Research Council of Canada. This research has made use of the SIMBAD database, operated at CDS, Strasbourg, France. 


\section{REFERENCES}

Alexander, J. B. 1967, MNRAS, 137, 41

Alexander, D. R., \& Ferguson, J. W. 1994, ApJ, 437, 879

Anders, E., \& Grevesse, N. 1989, Geochim. Cosmochim. Acta, 53, 197

Arribas, S., \& Martinez-Roger, C. 1989, A\&A, 215, 305

Artymowicz, P. 1992, PASP, 104, 769

Bahcall, J., \& Pinsonneault, M. H. 1992, Rev Mod Phys 64, 885.

Bahcall, J. 1989, Neutrino Astrophysics (Cambridge: Cambridge University Press), 115

Bakos, G. G., 1971, JRASC, 65, 222

Balachandran, S. 1990, ApJ, 354, 310

Baliunas, S. L., Henry, G. W., Donahue, R. A., Fekel, F. C., \& Soon, W. H. 1997, ApJ, 474, L119

Baliunas, S. L., Donahue, R. A., Soon, W., Gilliland, R., Soderblom, D. R. 1995, BASS, 186, 21.09

Barshay, S. S. \& Lewis, J. S. 1976, ARA\&A, 14, 81

Barnes, T. G., Evans, D. S., \& Moffett, T. J. 1978, MNRAS, 183, 285

Beckwith, S. V. W. \& Sargent, A. I. 1996, Science 383, 139

Black, D. 1997, ApJ, 49, L171

Blackwell, D. E., Petford, A. D., Arribus, S., Haddock, D. J., \& Selby, M. J. 1990, A\&A, 232, 396

Boesgaard, A. M., Lavery, R. J., 1986, ApJ, 309, 762

Boden, A., Milman, M., Unwin, S., Yu, J., Shao, M. 1996, BAAS189, 19.09 Abstract

Boden, A. F., Belle, G. T. van, et al. 1998 astro-ph/9804016 ApJ, in press

Böhm-Vitense, E. 1958, Zs. f. Ap., 46, 108

Borucki, W. J., Summers, A. L. 1984, Icarus, 58, 121

Boss, A. P. 1995, Science, 267, 360

Boss, A. P. 1996, Lunar \& Planetary Science, 27, 139

Bressan, A., Chiosi, C., \& Fagotto, F. 1994, ApJS, 94, 63

Brown, T. M., Kotak, R., Horner, S. D., Kennelly, E., Korzennik, S., Nisenson, P., and Noyes, R. W., 1998, ApJ, 484, L85

Brown, T. M., Kotak, R., Horner, S. D., Kennelly, E., Korzennik, S., Nisenson, P., and Noyes, R. W., 1998, ApJS, in press

Butler, R. P. \& Marcy, G. W. 1996, ApJ, 464, L153

Butler, R. P., Tinney, C., Pennry, A., Jones, H., \& Marcy, G. 1996, ApJ, 474, L115

Butler, R.P., et al. 1998 PATT 1998A Proposal.

Campbell, B. 1978, ApJ, 83, 11

Canuto, V. M. \& Mazzitelli, I. 1992, ApJ, 389, 724

Cayrel de Strobel, G. et al. 1997, AASS 124, 299

Chambers, J. E., Wetherill, G. W. \& Boss, A. P. 1996, Icarus, 119, 261 
Chiappini, C. \& Maciel, W. J. 1994, 288, 921

Christensen-Dalsgaard, J. \& Gough, D. O. 1998, The Observatory, 118, 25

Christensen-Dalsgaard, J., Gough, D. O., \& Thompson, M. J. 1991, ApJ, 378, 413

Cochran, W. D., Hatzes, A. P.,\& Hancock, J. 1995, ApJ, 380, L35

Cochran, W. D., Hatzes, A. P., Butler, R. P. \& Marcy, G. W. 1997, ApJ, 483, 457

Cochran, W. \& Hatzes, A., 1997, 29th DPS Meeting.

Colavita, M. M., Shao, M. 1994 "Indirect Planet Detection with Ground-Based Long-Baseline Interferometry." In Planetary Systems: Formation, Evolution, and Detection, ed. Burke, B. F., Rahe, J. H., Roettger, E. E., 385, Kluwer Academic

Cox, J. P. 1968, Principles of Stellar Structure, in two volumes (New York: Gordon and Breach)

Demarque, P., Guenther, D. B., \& Kim, Y.-C. 1997, ApJ, 474, 790

Drake, S. A., Pravdo, S. H., Angelini, L. \& Stern, R. 1998, ApJ, 115, 2122

Edmonds, P., Cram, L., Demarque, P., Guenther, D. B., \& Pinsonneault, M. H. 1992, ApJ, 394, 313

Edvardsson, B., Andersen, J., Gustafsson, B., Lambert, D. L., Nissen, P. E., and Tomkin, J. 1993, A\&A, 275,101

Edvardsson, B., Andersen, J., Gustafsson, B., Lambert, D. L., Nissen, P. E., and Tomkin, J. 1993, A\&AS, 102,603

Eggleton, P. \& Kiseleva, L. 1997, The 10th Cambridge Workshop on Cool Stars, Stellar Systems, and the Sun.

ESA, 1989, The Hipparcos and Tycho Catalogues, ESA SP-1200

Faulkner, J. 1972, ApJ, 173, 401

Favata, F., Micela, G., Sciortino, S. 1997, A\&A, 275, 101

Ford, E. B. \& Rasio, F. A. 1998 in preparation

Francois, P., Spite, M., Gillet, D., Gonzalez, J.-F., \& Spite, F. 1996, ApJ, 319, L13

Fuhrmann, K., Pfeiffer, M. J., Bernkopf, J. 1997, A\&A, 326, 1081

Gatewood, G.D., 1996, BAAS188, 40.11 Abstract

Gladman, B. 1993, Icarus, 106, 247

Glanz, J. 1998, Science, 279, 170

Goldreich, P. \& Keeley, D. A. 1977, ApJ, 211, 934

Goldreich, P. \& Soter, S. 1966, Icarus, 5, 375

Gonzales, G. 1998, A\&A, 334, 221

Gonzales, G. 1998, private communications

Gonzales, G. 1997, MNRAS, 285, 403

Gonzales, G. 1997, private communications

Goodman, J., \& Oh, S.P. 1997, ApJ, 486, 403

Goodman, J., \& Diskson, E.S. 1998 astro-ph/9801289)

Gratton, R. G., Carretta, E., Castelli, F. 1996, A\&A, 314, 191 
Gray, D. F. 1982, ApJ, 258, 201

Gray, D. F. \& Nagar, P. 1985, ApJ, 298, 756

Gray, D. F. 1986, PASP, 98, 319

Gray, D. F. 1995, PASP, 107, 120

Gray, D. F. 1998, Nature, 391, 153

Green, E., Demarque, P., King, C. R. 1987 The revised yale isochrones and luminosity functions. Yale Observatory

Guenther, D. B., Demarque, P., Kim, Y.-C., \& Pinsonneault, M. H. 1992, ApJ, 387, 372

Guenther, D. B., \& Demarque, P. 1993, ApJ, 405, 298

Guenther, D. B., \& Demarque, P. 1996, ApJ, 456, 798

Guenther, D. B., \& Demarque, P. 1997, ApJ, 484, 937

Guenther, D. B., Kim, Y.-C., \& Demarque, P. 1996, ApJ, 463, 382

Guillot, T., Burrows, A., Hubbard, W. B., Lunine, J. I., \& Saumon, D., 1996, ApJ, 459, L35

Hansen, B. 1998, Private communications

Hatzes, A. P., Cochran, W. D., Johns-Krull, C. M., 1997, ApJ, 478, 374

Hatzes, A. P., Cochran, W. D., \& Bakker, E. J. 1998, Nature, 391, 154

Hearnshaw, J. B., 1972, MNRAS, 77, 55

Hearnshaw, J. B., 1974, A\&A, 36, 191

Henry, G. W., Baliunas, S. L., Donahue, R. A., Soon, W. H., \& Saar, S. H. 1997, ApJ, 474, 503

Hoffleit D., \& Warren Jr., W. H. 1991, The Bright Star Catalogue, 5th Revised (Preliminary Version), ftp://cdsarc.u-strasbg.fr/cats/V/50

Holman, M., Touma, J., \& Tremaine, S. 1997, Nature, 386, 20

Horner, S., Brown, T., Kennelly, E., Jha, S., Korzennik, S., Krockenberger, M., Nisenson, P., \& Noyes, R. 1997, The 10th Cambridge Workshop on Cool Stars, Stellar Systems, and the Sun.

Hut, P. 1980, A\&A, 92, 167

Iglasias, C. A., \& Rogers F. J., 1996 ApJ, 464, 943

Ioannou, P. J. \& Lindzen, R. S. 1993, ApJ, 406, 252

Imbert, M. \& Prevot, 1998, A\&A, 334, L37

Izotov, Y., Thuan, T., Lipovetsky, V. 1997, AJS, 108, 1

Jeffery, C. S., Bailey, M. E., \& Chambers, J. E. 1997, The Observatory, 117, 224.

Katz, J. L. 1997, ApJ, 484, 862

Kim, Y.-C. \& Chan, K. L. 1998, ApJ, 496, L121

Kiseleva, L. \& Eggleton, P. 1997. The 10th Cambridge Workshop on Cool Stars, Stellar Systems, and the Sun.

Kudritzki, R. P. \& Reimers, D. 1978, A\&A, 70, 227 
Korzennik, S. G., Brown, T. M., Contos, A. R., Horner, S., Jha, S., Kennelly, T., Krockenberger, M., Nisenson, P, \& Noyes, R. W. 1997, The 10th Cambridge Workshop on Cool Star, Stellar Systems, and the Sun.

Kubat, J., Holmgren, D., \& Rentzsch-Holm, I., 1998, A\&A 332, 842

Kuroczkin, D. \& Wiszniewski, A. 1977, AcA 27, 145

Kurucz, R. 1991 in Stellar Atmospheres: Beyond Classical Models, ed. L. Crivellari, I. Hubney and D. G. Hummer (Dordrecht: Kluwer), 440

Lin, D. N. C., Bodenheimer, P. \& Richardson, D. C. 1996, Nature, 380, 606

Lissauer, J. J. 1993, ARA\&A, 31, 129

Livio, M. 1994, in Circumstellar Media in Late Stages of Stellar Evolution, ed. R. E. S. Clegg, I. R. Stevens, \& W. P. S. Meikle (Cambridge: Cambridge Univ. Press), 35

Lopez, R. J. G. \& Taoro, M. R. P. 1998, A\&A 334, 599

Lubow, S., Tout, C., \& Livio, M. 1997, ApJ, 484, 886L

Maddox, J. 1994, Nature, 372, 611

Maede, A. 1993, A\&A, 268, 833

Mannings, V. 1998, Nature 393, 117

Marcy, G. W. \& Butler, R. P. 1996, ApJ, 464, L147

Marcy, G. W., Butler, R. P., Williams, E., Bildsten, L., Graham, J., Ghez, A., \& Jernigan, F. 1997, ApJ, 481,926

Marcy, G. W. \& Butler, R. P. 1998, A\&AAR, 36, 57

Marcy, G. W. 1998, private communications

Marsakov, V. A., \& Shevelev, Y. G. 1995 Bull. Inf. CDS, 47, 13

Mayor, M. \& Queloz, D. 1995, Nature, 378, 355

Mayor, M., Queloz, D., Udry, \& Halbwachs. 1997, From Brown Dwarves to Planets, IAU Colloquium \#161

Mazeh, T., Krymolowski, Y., \& Rosenfeld, G. 1996, ApJ, 466, 415

McAlister, H. A., Mason, B. D., Hartkopf, W. I., Shara, M. M., 1993, AJ106, 1639

McWilliam, A. 1990, ApJS, 74, 1075

Mihalas, D., Daeppen, W., \& Hummer, D. G. 1988, ApJ, 331, 815

Moffett, T. J. \& Barnes, T. G. III. 1979, PASP, 91, 180

Murray, N., Hansen, B., Holman, M., \& Tremaine, S. 1998, Science, 279, 69

Noyes, R. W., Hartmann, L., Baliunas, S. L., Duncan, D. K., \& Vaughan, A. H. 1984, ApJ, 279,763

Noyes, R. W., Jha, S., Korzennik, S., Krockenberger, M., Nisenson, P., Brown, T., Kennelly, E., \& Horner, S. 1997, ApJ, 483, L111; erratum 487, L195

Oinas, V. 1974, ApJS, 27, 405

Oinas, V. 1977, A\&A, 61, 16

Pagel, B. E. J., Simonson, E. A., Terlevich, R. J., and Edmunds, M. G. 1992, MNRAS, 255, 325

Peimbert, M., 1986, PASP, 98, 1057 
Peimbert, M., Sarmiento, A.., and Colin, P. 1994, RMxAA, 28, 181

Perrin, M. N., Nejlesen, P. M., Cayrel de Strobel, G., \& Cayrel, R. 1977, A\&A, 54, 779

Perryman, M. A. C., et al., 1997, A\&A, 323, L49

Pols, O. R., Tout, C. A., Eggleton, P. P., \& Han, Z. 1995, MNRAS, 274, 964

Powell, A. L. T. 1970 MNRAS, 148, 477

Pravdo, S. H., Angelini, L., Drake, S. A., Stern, R. A., and White, N. E. 1996, New Astronomy, 1(2): 171

Rasio, F. A., \& Ford, E. 1996, Science, 274, 954

Rasio, F. A., Tout, C. A., Lubow, S. H. \& Livio, M. 1996, ApJ, 470, 1187

Reimers, D. 1975, in Problems in Stellar Atmospheres and Envelopes, ed. B. Bascheck, W. H. Kegal, \& G. Traving, (New York: Springer-Verlag), 229

Rogers, F. J. \& Iglesias, C. A. 1994, Science, 263, 50

Sackmann, I.-J., Boothroyd, A. I., \& Kraemer, K. E. 1993, ApJ, 418, 457

Skumanich, A. 1972, ApJ, 171, 565

Soderblom, D. R., Duncan, D. K., Johnson, D.R. 1991, ApJ, 375, 722

Soderblom, D. R. 1982, ApJ, 263, 239

Soderblom, D. R. 1983, ApJS, 53, 1

Spite, M. 1968, AnAp 31, 269

Spite M. \& Spite F. 1973, A\&A, 23, 63

Tassoul, J.-L. \& Tassoul, M. 1992, ApJ, 395, 259

Tassoul, M. \& Tassoul, J.-L. 1997, ApJ, 481, 363

Taylor, B. H. 1996, ApJS, 102, 105

Terquem, C., Papaloizou, J.C.B., Nelson, R.P., and Lin, D.N.C., 1997, Proceedings of the Xemes Rencontres de Blois "Planetary Systems: The Long View"

Thevenin, F., Vauclair, S., Vauclair, G., 1986, A\&A 166, 216

Tomkin, J., Edvardsson, B., Lambert, D. L., Gustafsson, B. 1997, A\&A, 327, 587

Tout C. A. \& Pringle, J. E. 1992, MNRAS, 256, 269

Trilling D., Benz, W., Guillot, T., Lunine, J. I., Hubbard, W. B., Burrows, A. 1998, ApJ, 500, 428

Unwin, S., Boden, A., Shao, M. 1996, BAAS189, 121.02 Abstract

Valenti, J. A., Butler, R. P. \& Marcy, G. W.. 1995 PASP, 107, 966

van Altena, W. F., Lee, T. J., \& Hoffleit, E. D. 1995, The General Catalogue of Trigonometric Parallaxes (4th ed.; New Haven: Yale Univ. Obs.)

Verbunt, F. \& Phinney, E. S. 1995, A\&A, 296, 709

Verbunt, F. \& Zwaan, C. 1981, A\&A, 100, L7

Wallerstein, G. 1962, ApJS, 6, 407

Weidemann, V. \& Koester, D. 1983, A\&A121, 77

Weidenschilling, S. \& Marzari, 1996, Nature, 384, 619 
Willems, B., Van Hoolst T., Smeyers, P., \& Waelkens, C., 1997, A\&A, 326, L37

Wolszczan, A. 1994, Science, 264, 538

Xu, Z., 1991, A\&A, 248, 367

Zahn, J.-P. 1977, A\&A, 57, 383; erratum 67, 162

Zahn, J.-P., \& Bouchet, L. 1989, A\&A, 223, 112

Zahn, J.-P. 1992 in "Binaries as Tracers of Stellar Formation," eds. A. Duquennoy \& M. Mayor (Cambridge U. Press), p. 253

Zahn, J.-P. 1994, A\&A, 288, 829 
Fig. 1. - This plot of planet mass versus orbital period indicates the regions allowed after imposing tidal stability constraints on 51 Pegasi. The lines labeled $\tau_{a}$ indicate where the timescale for orbital decay of the planet would be less than the age of the star, according to standard tidal dissipation theory. The lines labeled $\tau_{a}^{\prime}$ differ in that no reduction of the orbital decay timescale was applied to account for the long convective turnover timescale (Eq. 4). For both groups of lines labeled $\tau_{a}$ and $\tau_{a}^{\prime}$, the thicker line corresponds to our best model and the lines on either side reflect the uncertainty in the stellar age from our models (see Table 15). The lines labeled $K / \sin i$ indicate the typical detection limits of current radial velocity surveys. The line labeled $R_{L} / R_{J}$ indicates where a planet with mass $0.4 M_{\mathrm{J}}$, and radius $\simeq 1 R_{J} \simeq 0.1 R_{\odot}$, would overflow its Roche lobe. The large dot indicates the minimum mass of the planet. This plot was constructed using our best model parameters: $M_{*}=1.05 M_{\odot}, R_{*}=1.16 R_{\odot}, \tau_{c}=18.6 \mathrm{~d}, M_{\mathrm{ce}}=0.023 M_{\odot}$, and $\tau_{\mathrm{MS}}=7.6_{-5.1}^{+4.0}$ Gyr.

Fig. 2.- This plot shows several time scales as a function of the mass of the companion orbiting 51 Peg. Here $\tau_{\mathrm{a}}$ is the orbital decay timescale, $\tau_{\mathrm{su}}^{*}$ is the spin-up timescale for the whole star, and $\tau_{\mathrm{su}}^{\mathrm{ce}}$ is the spin-up timescale for the convective envelope alone. The dashed diagonal lines correspond to setting $f=1$, while the solid diagonal lines correspond to calculating $f$ from Eq. 4 with $f^{\prime}=1$. The solid horizontal line indicates the age of the system, $\tau_{\mathrm{MS}}$, as determined from our best model, while the dashed horizontal lines indicate the uncertainty in the age. The intersections of the age with the spin-up timescales gives the maximum companion masses quoted in the text.

Fig. 3.- This plot of planet mass versus orbital period indicates the regions allowed by tidal stability constraints on $\tau$ Boo. Here we have taken our model with the largest convective envelope. The model has $M_{*}=1.30 M_{\odot}, R_{*}=1.51 R_{\odot}, \tau_{c}=7.93 \mathrm{~d}, M_{\mathrm{ce}}=0.0035 M_{\odot}$, and an age of $2.9 \mathrm{Gyr}$. This model is for $T_{\text {eff }}=6200 \mathrm{~K},[\mathrm{Fe} / \mathrm{H}]=0.16$, and $L_{*}=3.006 L_{\odot}$. Other conventions are as in Fig. 1.

Fig. 4.- This plot shows several time scales as a function of the mass of the companion orbiting $\rho^{1}$ Cnc. The labels are the same as in Fig. 2. The intersection of the age with the timescales for tidal spin-up lead to the maximum companion masses listed in the text.

Fig. 5.- The solid line (left axis) shows the evolution of the Sun's convective envelope. The dashed lines (right axis) indicate the surface metallicity which would result from the instantaneous accretion of rocky material onto the star at each time in the Sun's past, assuming that the accreted material is mixed across the convective envelope. Starting from the bottom, the dashed lines correspond to accreting 5, 10, 25, 50, 100, 500, and $1000 M_{\oplus}$. We see that producing the high surface metallicities seen in $51 \mathrm{Peg}(0.21 \mathrm{dex})$ and $\rho^{1}$ Cnc $(0.29 \mathrm{dex})$ is possible with the accretion of a large amount of rocky material after $\sim 10^{7} \mathrm{yr}$. 
Table 1. Dynamical Properties of Extrasolar Planets ${ }^{\text {a }}$

\begin{tabular}{cccccccc}
\hline \hline Star & HD & $\begin{array}{c}M_{*} \\
\left(M_{\odot}\right)\end{array}$ & $\begin{array}{c}P_{\text {orb }} \\
\text { (days) }\end{array}$ & e & $\begin{array}{c}a \\
(\mathrm{AU})\end{array}$ & $\begin{array}{c}m \sin i \\
\left(M_{\mathrm{J}}\right)\end{array}$ & $\begin{array}{c}\text { Ref } \\
\end{array}$ \\
& & & & & & \\
\hline$\tau$ Boo & 120136 & $1.37 \pm 0.09$ & $3.3128 \pm 0.0002$ & $0.018 \pm 0.016$ & $0.0413 \pm 0.0010$ & $4.74 \pm 0.10$ & {$[1]$} \\
$51 \mathrm{Peg}$ & 217014 & $1.05 \pm 0.09$ & $4.2311 \pm 0.0005$ & $0.012 \pm 0.010$ & $0.0520 \pm 0.0015$ & $0.464 \pm 0.036$ & {$[2]$} \\
$v$ And & 9826 & $1.34 \pm 0.12$ & $4.611 \pm 0.005$ & $0.109 \pm 0.040$ & $0.0598 \pm 0.018$ & $0.739 \pm 0.075$ & {$[1]$} \\
$\rho^{1}$ Cnc & 75732 & $0.95 \pm 0.10$ & $14.648 \pm 0.0009$ & $0.051 \pm 0.013$ & $0.1152 \pm 0.0038$ & $0.874 \pm 0.079$ & {$[1]$} \\
$\rho$ CrB & 143761 & $0.89 \pm 0.05$ & $39.645 \pm 0.088$ & $0.15 \pm 0.03$ & $0.2192 \pm 0.0042$ & $1.064 \pm 0.059$ & {$[3,7]$} \\
HD 114762 & 114762 & $0.75 \pm 0.15$ & $83.90 \pm 0.08$ & $0.35 \pm 0.05$ & $0.34 \pm 0.04$ & $10.5 \pm 1.9$ & {$[4]$} \\
$70 \mathrm{Vir}$ & 117176 & $1.01_{-0.02}^{+0.05}$ & $116.67 \pm 0.01$ & $0.4 \pm 0.01$ & $0.47 \pm 0.01$ & $7.3 \pm 0.3$ & {$[5]$} \\
$16 \mathrm{Cyg} \mathrm{B}$ & 186427 & $0.96 \pm 0.05$ & $800.8 \pm 11.7$ & $0.634 \pm 0.082$ & $1.66 \pm 0.05$ & $2.0 \pm 0.3$ & {$[6]$} \\
$47 \mathrm{UMa}$ & 95128 & $1.01 \pm 0.05$ & $1090 \pm 15$ & $0.03 \pm 0.06$ & $2.08 \pm 0.06$ & $2.3 \pm 0.2$ & {$[5]$} \\
\hline
\end{tabular}

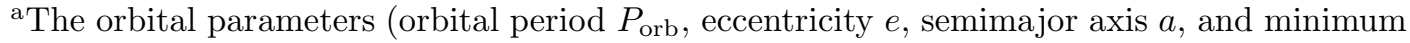
mass $m \sin i$ ) are from: [1] Butler \& Marcy 1996; [2] Marcy et al. 1997; [3] Noyes et al. 1997; [4] Mazeh et al. 1996; [5] Marcy \& Butler 1996; [6] Cochran et al. 1997a; [7] Marcy 1998. In order to determine $a$ and $m \sin i$, we have assumed the stellar mass given in column 3 . The first five stellar masses are based on the results of this paper and are discussed later. The remaining stellar masses are based on preliminary calculations which will be presented in a subsequent paper. 
Table 2. Observed Parameters of Stars with Extrasolar Planets ${ }^{b}$

\begin{tabular}{cccccccc}
\hline \hline Star & MK & $M_{V}$ & $T_{\text {eff }}(\mathrm{K})$ & {$[\mathrm{Fe} / \mathrm{H}]$} & $\log \mathrm{g}(\mathrm{cgs})$ & $\mathrm{BC}(\mathrm{V})$ & $L_{*} / L_{\odot}$ \\
\hline$\tau$ Boo & F7V & $3.535 \pm 0.024$ & $6550 \pm 100$ & $+0.34 \pm 0.09$ & $4.50 \pm 0.15$ & +0.02 & $3.006 \pm 0.213$ \\
51 Peg & G5V & $4.518 \pm 0.025$ & $5750 \pm 75$ & $+0.21 \pm 0.06$ & $4.40 \pm 0.10$ & -0.07 & $1.321 \pm 0.094$ \\
$v$ And & F8V & $3.453 \pm 0.021$ & $6250 \pm 100$ & $+0.17 \pm 0.08$ & $4.30 \pm 0.10$ & -0.01 & $3.333 \pm 0.225$ \\
$\rho^{1}$ Cnc & G8V & $5.470 \pm 0.023$ & $5250 \pm 70$ & $+0.45 \pm 0.03$ & $4.40 \pm 0.15$ & -0.18 & $0.608 \pm 0.042$ \\
$\rho$ CrB & G2V & $4.184 \pm 0.027$ & $5750 \pm 75$ & $-0.29 \pm 0.06$ & $4.10 \pm 0.05$ & -0.07 & $1.796 \pm 0.132$ \\
HD 114762 & F9V & $4.259 \pm 0.123$ & $5950 \pm 75$ & $-0.60 \pm 0.06$ & $4.45 \pm 0.05$ & -0.07 & $1.676 \pm 0.290$ \\
70 Vir & G5V & $3.680 \pm 0.029$ & $5500 \pm 75$ & $-0.03 \pm 0.06$ & $3.90 \pm 0.10$ & -0.07 & $2.856 \pm 0.076$ \\
16 Cyg B & G5V & $4.597 \pm 0.024$ & $5700 \pm 75$ & $+0.06 \pm 0.06$ & $4.35 \pm 0.05$ & -0.07 & $1.228 \pm 0.087$ \\
4 UMa & G0V & $4.288 \pm 0.020$ & $5800 \pm 75$ & $+0.01 \pm 0.06$ & $4.25 \pm 0.05$ & -0.05 & $1.603 \pm 0.106$ \\
\hline
\end{tabular}

${ }^{\mathrm{b}}$ Spectral type from Hipparcos Catalogue; $M_{V}$ derived from Hipparcos Catalogue (ESA, 1997) photometric and parallax data; $T_{\text {eff }},[\mathrm{Fe} / \mathrm{H}]$, and $\log g$ from Gonzalez 1997ab, 1998ab; $\mathrm{BC}(\mathrm{V})$ derived from previous parameters using color calibration as in the Yale isochrones (Green et al. 1987); $L_{*} / L_{\odot}$ calculated from the Hipparcos absolute visual magnitude and $\mathrm{BC}(\mathrm{V})$. 
Table 3. Primary Results of Stellar Model Calculations

\begin{tabular}{|c|c|c|c|c|c|c|c|c|c|}
\hline Star & $\begin{array}{c}M_{*} \\
\left(M_{\odot}\right)\end{array}$ & $\begin{array}{c}R_{*} \\
\left(R_{\odot}\right)\end{array}$ & $\begin{array}{l}\text { Age } \\
\text { (GYr) }\end{array}$ & $\begin{array}{c}M_{\mathrm{ce}} \\
\left(M_{\odot}\right)\end{array}$ & $\begin{array}{c}R_{\mathrm{ce}} \\
\left(R_{\odot}\right)\end{array}$ & $\begin{array}{l}\tau_{c} \\
\text { (d) }\end{array}$ & $k_{*}^{2}$ & $k_{\mathrm{ce}}^{2}$ & $\begin{array}{c}M_{\mathrm{ce}, \mathrm{ZAMS}} \\
\left(M_{\odot}\right)\end{array}$ \\
\hline$\tau$ Boo & $1.37 \pm 0.08$ & $1.41_{-0.09}^{+0.10}$ & $1.2_{-0.8}^{+1.2}$ & $\lesssim 0.002$ & $1.19 \pm 0.09$ & $\lesssim 5.8$ & 0.10 & $\lesssim 0.002$ & 0.008 \\
\hline $51 \mathrm{Peg}$ & $1.05_{-0.08}^{+0.09}$ & $1.16 \pm 0.07$ & $7.6_{-5.1}^{+4.0}$ & $0.023_{-0.006}^{+0.007}$ & $0.82 \pm 0.04$ & $18.6 \pm 2.5$ & 0.12 & 0.020 & 0.037 \\
\hline$v$ And & $1.34_{-0.12}^{+0.07}$ & $1.56_{-0.10}^{+0.11}$ & $2.6_{-1.0}^{+2.1}$ & $0.002_{-0.002}^{+0.003}$ & $1.29_{-0.06}^{+0.07}$ & $6.8_{-6.8}^{+2.3}$ & 0.10 & 0.002 & 0.006 \\
\hline$\rho^{1} \mathrm{Cnc}$ & $0.95_{-0.09}^{+0.11}$ & $0.93_{-0.03}^{+0.02}$ & $8.4_{-8.3}^{+7.1}$ & $0.046_{-0.006}^{+0.004}$ & $0.63 \pm 0.03$ & $26.7_{-2.2}^{+1.2}$ & 0.15 & 0.048 & 0.069 \\
\hline$\rho \mathrm{CrB}$ & $0.89_{-0.04}^{+0.05}$ & $1.35_{-0.08}^{+0.09}$ & $14.1_{-2.4}^{+2.0}$ & $0.033_{-0.009}^{+0.011}$ & $0.88_{-0.03}^{+0.05}$ & $21.5_{-2.8}^{+2.9}$ & 0.10 & 0.021 & 0.029 \\
\hline
\end{tabular}


Table 4. Summary of Observational Data for $51 \mathrm{Peg}$

\begin{tabular}{|c|c|c|c|c|}
\hline$T_{\text {eff }}(\mathrm{K})$ & {$[\mathrm{Fe} / \mathrm{H}]($ solar $)$} & $\log g(\operatorname{cgs})$ & $v \sin i\left(\mathrm{~km} \mathrm{~s}^{-1}\right)$ & Reference \\
\hline $5750 \pm 75$ & $0.22 \pm 0.06$ & $4.40 \pm 0.10$ & $1.4 \pm 0.3$ & Gonzalez 1997a, 1998 \\
\hline \multirow[t]{2}{*}{$5793 \pm 70$} & $0.20 \pm 0.07$ & $4.33 \pm 0.10$ & $2.0 \pm 1.0$ & Fuhrmann et al. 1997 \\
\hline & & & $2.35 \pm 0.1$ & Hatzes et al. 1997 \\
\hline \multirow[t]{2}{*}{5775} & $0.20 \pm 0.07$ & 4.35 & & Tomkin et al. 1997 \\
\hline & & & $2.4 \pm 0.3$ & Francois et al. 1996 \\
\hline \multirow[t]{3}{*}{5669} & & 4.06 & & Gratton et al. 1996 \\
\hline & $0.172 \pm 0.049$ & & & Taylor $1996^{\mathrm{c}}$ \\
\hline & 0.19 & 4.32 & $2.8 \pm 0.5$ & Mayor \& Queloz 1995 \\
\hline 5730 & & & & Gray 1995 \\
\hline 5773 & & & & Grennon (cited by Mayor \& Queloz 1995) \\
\hline 5724 & & 4.30 & & Valenti (cited by Mayor \& Queloz 1995) \\
\hline 5755 & 0.06 & 4.18 & & Edvardsson 1993b \\
\hline \multirow[t]{2}{*}{5740} & 0.05 & 3.76 & & McWilliam 1990 \\
\hline & & 4.13 & & Xu 1991 \\
\hline 5750 & 0.12 & & $1.7 \pm 0.8$ & Soderblom 1982 \\
\hline 5727 & 0.12 & 4.27 & & Hearnshaw 1972 \\
\hline
\end{tabular}

${ }^{c}$ This value is based on a statistical analysis of previous measurements and not any new observations. 
Table 5. Stellar Models for $51 \mathrm{Peg}$

\begin{tabular}{|c|c|c|c|c|c|c|c|c|c|c|c|c|c|c|c|}
\hline $\begin{array}{c}M_{*} \\
\text { Solar }\end{array}$ & $\begin{array}{l}{\left[\frac{F e}{H}\right]} \\
\text { Solar }\end{array}$ & $\begin{array}{c}T_{\text {eff }} \\
\mathrm{K}\end{array}$ & $\begin{array}{c}L_{*} \\
\text { Solar }\end{array}$ & $\mathrm{Z}$ & $\mathrm{X}$ & $\mathrm{Y}$ & $\begin{array}{l}\text { Age } \\
\text { Gyr }\end{array}$ & $\begin{array}{c}M_{c e} \\
\text { Solar }\end{array}$ & $\begin{array}{l}\text { PSH } \\
\text { Solar }\end{array}$ & $\begin{array}{c}R_{c e} \\
\text { Solar }\end{array}$ & $\begin{array}{c}L_{*} \\
\text { Solar }\end{array}$ & $\begin{array}{c}R_{*} \\
\text { Solar }\end{array}$ & $\begin{array}{c}T_{\text {eff }} \\
\mathrm{K}\end{array}$ & $\begin{array}{c}\log g \\
\operatorname{cgs}\end{array}$ & $\begin{array}{c}\frac{\delta Y}{\delta Z} \\
\text { Solar }\end{array}$ \\
\hline 1.092 & 0.21 & 5750 & 1.321 & 0.030 & 0.687 & 0.283 & 6.4 & 0.02226 & 0.0942 & 0.835 & 1.322 & 1.161 & 5751 & 4.35 & 0.000 \\
\hline 1.067 & 0.21 & 5750 & 1.321 & 0.030 & 0.669 & 0.301 & 7.2 & 0.02313 & 0.0954 & 0.827 & 1.321 & 1.161 & 5750 & 4.34 & 1.500 \\
\hline 1.050 & 0.21 & 5750 & 1.321 & 0.030 & 0.657 & 0.312 & 7.6 & 0.02371 & 0.0962 & 0.822 & 1.321 & 1.161 & 5750 & 4.33 & 2.500 \\
\hline 1.033 & 0.21 & 5750 & 1.321 & 0.030 & 0.646 & 0.324 & 8.1 & 0.02429 & 0.0970 & 0.816 & 1.320 & 1.161 & 5749 & 4.32 & 3.500 \\
\hline 1.059 & 0.21 & 5675 & 1.321 & 0.030 & 0.687 & 0.283 & 8.8 & 0.02772 & 0.1013 & 0.833 & 1.321 & 1.192 & 5675 & 4.31 & 0.000 \\
\hline 1.033 & 0.21 & 5675 & 1.321 & 0.030 & 0.669 & 0.301 & 9.5 & 0.02856 & 0.1024 & 0.824 & 1.321 & 1.192 & 5675 & 4.30 & 1.500 \\
\hline 1.016 & 0.21 & 5675 & 1.321 & 0.030 & 0.657 & 0.312 & 10.1 & 0.02912 & 0.1031 & 0.819 & 1.321 & 1.192 & 5675 & 4.29 & 2.500 \\
\hline 0.998 & 0.21 & 5675 & 1.321 & 0.030 & 0.646 & 0.324 & 10.6 & 0.02968 & 0.1039 & 0.813 & 1.321 & 1.192 & 5675 & 4.28 & 3.500 \\
\hline 1.124 & 0.21 & 5825 & 1.321 & 0.030 & 0.687 & 0.283 & 4.2 & 0.01769 & 0.0875 & 0.836 & 1.324 & 1.132 & 5828 & 4.38 & 0.000 \\
\hline 1.100 & 0.21 & 5825 & 1.321 & 0.030 & 0.669 & 0.301 & 4.9 & 0.01843 & 0.0886 & 0.829 & 1.324 & 1.132 & 5827 & 4.37 & 1.500 \\
\hline 1.084 & 0.21 & 5825 & 1.321 & 0.030 & 0.657 & 0.312 & 5.3 & 0.01893 & 0.0893 & 0.824 & 1.323 & 1.132 & 5827 & 4.37 & 2.500 \\
\hline 1.068 & 0.21 & 5825 & 1.321 & 0.030 & 0.646 & 0.324 & 5.8 & 0.01942 & 0.0901 & 0.819 & 1.323 & 1.132 & 5826 & 4.36 & 3.500 \\
\hline 1.050 & 0.15 & 5750 & 1.321 & 0.027 & 0.690 & 0.283 & 7.8 & 0.02328 & 0.0960 & 0.825 & 1.325 & 1.161 & 5755 & 4.33 & 0.000 \\
\hline 1.032 & 0.15 & 5750 & 1.321 & 0.027 & 0.679 & 0.295 & 8.4 & 0.02390 & 0.0968 & 0.819 & 1.324 & 1.161 & 5753 & 4.32 & 1.500 \\
\hline 1.020 & 0.15 & 5750 & 1.321 & 0.027 & 0.671 & 0.302 & 8.8 & 0.02431 & 0.0974 & 0.815 & 1.323 & 1.161 & 5752 & 4.32 & 2.500 \\
\hline 1.008 & 0.15 & 5750 & 1.321 & 0.027 & 0.663 & 0.310 & 9.2 & 0.02472 & 0.0980 & 0.811 & 1.322 & 1.161 & 5751 & 4.31 & 3.500 \\
\hline 1.099 & 0.27 & 5750 & 1.321 & 0.035 & 0.658 & 0.307 & 6.0 & 0.02224 & 0.0938 & 0.835 & 1.321 & 1.161 & 5750 & 4.35 & 1.500 \\
\hline 1.073 & 0.27 & 5750 & 1.321 & 0.035 & 0.641 & 0.324 & 6.7 & 0.02294 & 0.0950 & 0.828 & 1.321 & 1.161 & 5750 & 4.34 & 2.500 \\
\hline 1.048 & 0.27 & 5750 & 1.321 & 0.035 & 0.625 & 0.340 & 7.5 & 0.02364 & 0.0962 & 0.820 & 1.321 & 1.161 & 5749 & 4.33 & 3.500 \\
\hline 1.092 & 0.21 & 5750 & 1.233 & 0.030 & 0.687 & 0.283 & 5.6 & 0.02203 & 0.0902 & 0.812 & 1.234 & 1.122 & 5751 & 4.38 & 0.000 \\
\hline 1.068 & 0.21 & 5750 & 1.233 & 0.030 & 0.669 & 0.301 & 6.3 & 0.02287 & 0.0913 & 0.804 & 1.233 & 1.122 & 5750 & 4.37 & 1.500 \\
\hline 1.052 & 0.21 & 5750 & 1.233 & 0.030 & 0.657 & 0.312 & 6.8 & 0.02343 & 0.0920 & 0.799 & 1.233 & 1.122 & 5750 & 4.36 & 2.500 \\
\hline 1.036 & 0.21 & 5750 & 1.233 & 0.030 & 0.646 & 0.324 & 7.3 & 0.02400 & 0.0928 & 0.794 & 1.233 & 1.122 & 5750 & 4.35 & 3.500 \\
\hline 1.094 & 0.21 & 5750 & 1.415 & 0.030 & 0.687 & 0.283 & 7.1 & 0.02251 & 0.0982 & 0.860 & 1.415 & 1.202 & 5750 & 4.32 & 0.000 \\
\hline 1.068 & 0.21 & 5750 & 1.415 & 0.030 & 0.669 & 0.301 & 7.8 & 0.02328 & 0.0994 & 0.852 & 1.415 & 1.202 & 5750 & 4.31 & 1.500 \\
\hline 1.050 & 0.21 & 5750 & 1.415 & 0.030 & 0.657 & 0.312 & 8.2 & 0.02379 & 0.1002 & 0.846 & 1.415 & 1.202 & 5750 & 4.30 & 2.500 \\
\hline 1.033 & 0.21 & 5750 & 1.415 & 0.030 & 0.646 & 0.324 & 8.7 & 0.02431 & 0.1010 & 0.841 & 1.415 & 1.202 & 5750 & 4.29 & 3.500 \\
\hline
\end{tabular}


Table 6. Summary of Observational Data for $\tau$ Boo

\begin{tabular}{ccccl}
\hline \hline$T_{\text {eff }}(\mathrm{K})$ & {$[\mathrm{Fe} / \mathrm{H}](\mathrm{solar})$} & $\log g(\mathrm{cgs})$ & $v \sin i\left(\mathrm{~km} \mathrm{~s}^{-1}\right)$ & \multicolumn{1}{c}{ Reference } \\
\hline $6360 \pm 80$ & $0.27 \pm 0.08$ & $4.17 \pm 0.10$ & $15.6 \pm 0.7$ & $\begin{array}{l}\text { Fuhrmann et al. 1998 } \\
\text { Gonzalez 1997b (spectroscopic upon additional analysis) } \\
6550 \pm 100\end{array}$ \\
$6600 \pm 100$ & $0.34 \pm 0.09$ & $4.50 \pm 0.15$ & $14.5 \pm 0.5$ & $\begin{array}{l}\text { Gonzalez 1997a, 1998 (spectroscopic) } \\
\text { Gonzalez 1997a (photometric) }\end{array}$ \\
6405 & 0.18 & & & Baliunas et al. 1997 \\
& & & Marsakov et al. 1995 \\
6358 & 0.09 & 4.22 & & Boesgaard \& Lavery 1986 \\
6390 & 0.30 & 3.8 & & Thevenin et al. 1986 \\
6460 & 0.00 & 4.3 & & Thevenin \& Foy 1983 \\
6300 & 0.00 & 4.60 & & Gray 1982 \\
6490 & 0.21 & & Soderblom 1982 \\
6380 & 0.14 & 4.3 & & Kuroczkin \& Wiszniewski 1977 \\
6450 & 0.28 & 4.3 & & Perrin 1977 \\
6462 & 0.28 & 4.3 & & Spite 1968 \\
\hline \hline
\end{tabular}


Table 7. Stellar Models for $\tau$ Boo

\begin{tabular}{|c|c|c|c|c|c|c|c|c|c|c|c|c|c|c|c|}
\hline $\begin{array}{c}M_{*} \\
\text { Solar }\end{array}$ & $\begin{array}{l}{\left[\frac{F e}{H}\right]} \\
\text { Solar }\end{array}$ & $\begin{array}{c}T_{\text {eff }} \\
\mathrm{K}\end{array}$ & $\begin{array}{c}L_{*} \\
\text { Solar }\end{array}$ & $\mathrm{Z}$ & $\mathrm{X}$ & $\mathrm{Y}$ & $\begin{array}{l}\text { Age } \\
\text { Gyr }\end{array}$ & $\begin{array}{c}M_{c e} \\
\text { Solar }\end{array}$ & $\begin{array}{c}\text { PSH } \\
\text { Solar }\end{array}$ & $\begin{array}{c}R_{c e} \\
\text { Solar }\end{array}$ & $\begin{array}{c}L_{*} \\
\text { Solar }\end{array}$ & $\begin{array}{c}R_{*} \\
\text { Solar }\end{array}$ & $\begin{array}{c}T_{\text {eff }} \\
\mathrm{K}\end{array}$ & $\begin{array}{c}\log g \\
\operatorname{cgs}\end{array}$ & $\begin{array}{c}\frac{\delta Y}{\delta Z} \\
\text { Solar }\end{array}$ \\
\hline 1.403 & 0.25 & 6400 & 2.808 & 0.033 & 0.684 & 0.283 & 0.5 & 0.00000 & 0.0000 & 1.154 & 2.816 & 1.367 & 6404 & 4.31 & 0.000 \\
\hline 1.375 & 0.25 & 6400 & 2.808 & 0.033 & 0.662 & 0.305 & 0.7 & 0.00000 & 0.0000 & 1.157 & 2.812 & 1.367 & 6402 & 4.30 & 1.500 \\
\hline 1.357 & 0.25 & 6400 & 2.808 & 0.033 & 0.647 & 0.320 & 0.9 & 0.00000 & 0.0000 & 1.159 & 2.809 & 1.367 & 6401 & 4.30 & 2.500 \\
\hline 1.338 & 0.25 & 6400 & 2.808 & 0.033 & 0.632 & 0.334 & 1.1 & 0.00000 & 0.0000 & 1.161 & 2.807 & 1.366 & 6399 & 4.29 & 3.500 \\
\hline 1.350 & 0.16 & 6400 & 3.006 & 0.027 & 0.690 & 0.283 & 1.5 & 0.00000 & 0.0000 & 1.191 & 3.008 & 1.414 & 6401 & 4.27 & 0.000 \\
\hline 1.337 & 0.16 & 6400 & 3.006 & 0.027 & 0.677 & 0.296 & 1.6 & 0.00000 & 0.0000 & 1.193 & 3.007 & 1.414 & 6400 & 4.26 & 1.500 \\
\hline 1.329 & 0.16 & 6400 & 3.006 & 0.027 & 0.669 & 0.304 & 1.7 & 0.00000 & 0.0000 & 1.194 & 3.007 & 1.414 & 6400 & 4.26 & 2.500 \\
\hline 1.320 & 0.16 & 6400 & 3.006 & 0.027 & 0.661 & 0.312 & 1.7 & 0.00000 & 0.0000 & 1.196 & 3.007 & 1.414 & 6400 & 4.26 & 3.500 \\
\hline 1.450 & 0.34 & 6350 & 3.006 & 0.041 & 0.676 & 0.283 & 0.8 & 0.00000 & 0.0000 & 1.230 & 3.015 & 1.437 & 6353 & 4.28 & 0.000 \\
\hline 1.416 & 0.34 & 6350 & 3.006 & 0.041 & 0.642 & 0.316 & 1.0 & 0.00000 & 0.0000 & 1.224 & 3.013 & 1.437 & 6353 & 4.27 & 1.500 \\
\hline 1.392 & 0.34 & 6350 & 3.006 & 0.041 & 0.620 & 0.339 & 1.1 & 0.00024 & 0.0112 & 1.220 & 3.012 & 1.437 & 6353 & 4.27 & 2.500 \\
\hline 1.369 & 0.34 & 6350 & 3.006 & 0.041 & 0.598 & 0.361 & 1.3 & 0.00096 & 0.0452 & 1.215 & 3.011 & 1.436 & 6352 & 4.26 & 3.500 \\
\hline 1.383 & 0.25 & 6300 & 3.006 & 0.033 & 0.684 & 0.283 & 1.7 & 0.00140 & 0.0729 & 1.241 & 3.013 & 1.460 & 6302 & 4.25 & 0.000 \\
\hline 1.362 & 0.25 & 6300 & 3.006 & 0.033 & 0.662 & 0.305 & 1.8 & 0.00163 & 0.0750 & 1.232 & 3.013 & 1.460 & 6302 & 4.24 & 1.500 \\
\hline 1.349 & 0.25 & 6300 & 3.006 & 0.033 & 0.647 & 0.320 & 1.9 & 0.00178 & 0.0763 & 1.227 & 3.013 & 1.460 & 6303 & 4.24 & 2.500 \\
\hline 1.335 & 0.25 & 6300 & 3.006 & 0.033 & 0.632 & 0.334 & 2.0 & 0.00193 & 0.0777 & 1.221 & 3.013 & 1.459 & 6303 & 4.23 & 3.500 \\
\hline 1.423 & 0.25 & 6450 & 3.006 & 0.033 & 0.684 & 0.283 & 0.5 & 0.00000 & 0.0000 & 1.159 & 3.008 & 1.392 & 6451 & 4.30 & 0.000 \\
\hline 1.397 & 0.25 & 6450 & 3.006 & 0.033 & 0.662 & 0.305 & 0.7 & 0.00000 & 0.0000 & 1.162 & 3.008 & 1.392 & 6451 & 4.30 & 1.500 \\
\hline 1.380 & 0.25 & 6450 & 3.006 & 0.033 & 0.647 & 0.320 & 0.8 & 0.00000 & 0.0000 & 1.164 & 3.008 & 1.392 & 6451 & 4.29 & 2.500 \\
\hline 1.363 & 0.25 & 6450 & 3.006 & 0.033 & 0.632 & 0.334 & 1.0 & 0.00000 & 0.0000 & 1.166 & 3.008 & 1.392 & 6451 & 4.28 & 3.500 \\
\hline 1.408 & 0.25 & 6400 & 3.006 & 0.033 & 0.684 & 0.283 & 0.9 & 0.00000 & 0.0000 & 1.189 & 3.005 & 1.414 & 6400 & 4.29 & 0.000 \\
\hline 1.384 & 0.25 & 6400 & 3.006 & 0.033 & 0.662 & 0.305 & 1.1 & 0.00000 & 0.0000 & 1.192 & 3.006 & 1.414 & 6400 & 4.28 & 1.500 \\
\hline 1.368 & 0.25 & 6400 & 3.006 & 0.033 & 0.647 & 0.320 & 1.2 & 0.00000 & 0.0000 & 1.194 & 3.007 & 1.414 & 6400 & 4.27 & 2.500 \\
\hline 1.351 & 0.25 & 6400 & 3.006 & 0.033 & 0.632 & 0.334 & 1.3 & 0.00000 & 0.0000 & 1.196 & 3.008 & 1.414 & 6401 & 4.27 & 3.500 \\
\hline 1.419 & 0.25 & 6400 & 3.218 & 0.033 & 0.684 & 0.283 & 1.2 & 0.00000 & 0.0000 & 1.224 & 3.220 & 1.463 & 6401 & 4.26 & 0.000 \\
\hline 1.396 & 0.25 & 6400 & 3.218 & 0.033 & 0.662 & 0.305 & 1.3 & 0.00000 & 0.0000 & 1.228 & 3.218 & 1.463 & 6400 & 4.25 & 1.500 \\
\hline 1.381 & 0.25 & 6400 & 3.218 & 0.033 & 0.647 & 0.320 & 1.4 & 0.00000 & 0.0000 & 1.230 & 3.216 & 1.463 & 6399 & 4.25 & 2.500 \\
\hline 1.366 & 0.25 & 6400 & 3.218 & 0.033 & 0.632 & 0.334 & 1.5 & 0.00000 & 0.0000 & 1.233 & 3.215 & 1.463 & 6398 & 4.24 & 3.500 \\
\hline
\end{tabular}


Table 8. Summary of Observational Data for $v$ And

\begin{tabular}{ccccl}
\hline \hline$T_{\text {eff }}(\mathrm{K})$ & {$[\mathrm{Fe} / \mathrm{H}](\mathrm{solar})$} & $\log g(\mathrm{cgs})$ & $v \sin i\left(\mathrm{~km} \mathrm{~s}^{-1}\right)$ & \multicolumn{1}{c}{ Reference } \\
\hline $6107 \pm 80$ & $0.09 \pm 0.06$ & $4.01 \pm 0.10$ & $9.5 \pm 0.8$ & Fuhrmann et al. 1998 \\
$6250 \pm 100$ & $0.17 \pm 0.08$ & $4.30 \pm 0.10$ & $9.0 \pm 0.5$ & Gonzalez 1997a, 1998 \\
6125 & 0.06 & 3.98 & & Gratton et al. 1996 \\
6187 & -0.02 & 4.13 & & Marsakov et al. 1995 \\
6205 & & & Blackwell et al. 1994 \\
6212 & 0.09 & 4.17 & & Edvardsson et al. 1993b \\
6198 & -0.03 & 4.22 & & Balachandran 1990 \\
6050 & 0.06 & 4.0 & & Boesgaard \& Lavery 1986 \\
& & & $9.0 \pm 0.4$ & Gray 1986 \\
6146 & -0.20 & 4.60 & & Thevenin \& Foy 1983 \\
6000 & -0.23 & 3.91 & & Hearnshaw 1974 \\
6072 & -0.14 & 4.10 & & Spite \& Spite 1973 \\
6072 & -0.11 & & Powell 1970 \\
\hline \hline
\end{tabular}


Table 9. Stellar Models for $v$ And

\begin{tabular}{|c|c|c|c|c|c|c|c|c|c|c|c|c|c|c|c|}
\hline $\begin{array}{c}M_{*} \\
\text { Solar }\end{array}$ & $\begin{array}{l}{\left[\frac{F e}{H}\right]} \\
\text { Solar }\end{array}$ & $\begin{array}{c}T_{\text {eff }} \\
\mathrm{K}\end{array}$ & $\begin{array}{c}L_{*} \\
\text { Solar }\end{array}$ & Z & $\mathrm{X}$ & Y & $\begin{array}{l}\text { Age } \\
\text { Gyr }\end{array}$ & $\begin{array}{c}M_{c e} \\
\text { Solar }\end{array}$ & $\begin{array}{l}\text { PSH } \\
\text { Solar }\end{array}$ & $\begin{array}{c}R_{c e} \\
\text { Solar }\end{array}$ & $\begin{array}{c}L_{*} \\
\text { Solar }\end{array}$ & $\begin{array}{c}R_{*} \\
\text { Solar }\end{array}$ & $\begin{array}{c}T_{\text {eff }} \\
\mathrm{K}\end{array}$ & $\begin{array}{l}\log g \\
\operatorname{cgs}\end{array}$ & $\begin{array}{c}\frac{\delta Y}{\delta Z} \\
\text { Solar }\end{array}$ \\
\hline 1.359 & 0.17 & 6250 & 3.333 & 0.028 & 0.689 & 0.283 & 2.5 & 0.00204 & 0.0841 & 1.304 & 3.336 & 1.561 & 6252 & 4.18 & 0.000 \\
\hline 1.347 & 0.17 & 6250 & 3.333 & 0.028 & 0.676 & 0.297 & 2.5 & 0.00224 & 0.0856 & 1.297 & 3.334 & 1.561 & 6250 & 4.18 & 1.500 \\
\hline 1.339 & 0.17 & 6250 & 3.333 & 0.028 & 0.667 & 0.306 & 2.6 & 0.00238 & 0.0866 & 1.293 & 3.332 & 1.561 & 6250 & 4.18 & 2.500 \\
\hline 1.331 & 0.17 & 6250 & 3.333 & 0.028 & 0.658 & 0.315 & 2.6 & 0.00251 & 0.0876 & 1.289 & 3.330 & 1.561 & 6249 & 4.17 & 3.500 \\
\hline 1.353 & 0.17 & 6150 & 3.333 & 0.028 & 0.689 & 0.283 & 2.9 & 0.00376 & 0.0964 & 1.310 & 3.335 & 1.613 & 6150 & 4.15 & 0.000 \\
\hline 1.339 & 0.17 & 6150 & 3.333 & 0.028 & 0.676 & 0.297 & 3.0 & 0.00406 & 0.0979 & 1.303 & 3.332 & 1.613 & 6149 & 4.15 & 1.500 \\
\hline 1.330 & 0.17 & 6150 & 3.333 & 0.028 & 0.667 & 0.306 & 3.0 & 0.00426 & 0.0989 & 1.299 & 3.330 & 1.612 & 6148 & 4.15 & 2.500 \\
\hline 1.320 & 0.17 & 6150 & 3.333 & 0.028 & 0.658 & 0.315 & 3.1 & 0.00445 & 0.0998 & 1.294 & 3.327 & 1.612 & 6147 & 4.14 & 3.500 \\
\hline 1.370 & 0.17 & 6350 & 3.333 & 0.028 & 0.689 & 0.283 & 2.0 & 0.00000 & 0.0000 & 1.291 & 3.340 & 1.513 & 6353 & 4.22 & 0.000 \\
\hline 1.357 & 0.17 & 6350 & 3.333 & 0.028 & 0.676 & 0.297 & 2.1 & 0.00000 & 0.0000 & 1.286 & 3.337 & 1.513 & 6352 & 4.21 & 1.500 \\
\hline 1.349 & 0.17 & 6350 & 3.333 & 0.028 & 0.667 & 0.306 & 2.1 & 0.00003 & 0.0012 & 1.283 & 3.336 & 1.513 & 6351 & 4.21 & 2.500 \\
\hline 1.341 & 0.17 & 6350 & 3.333 & 0.028 & 0.658 & 0.315 & 2.1 & 0.00031 & 0.0144 & 1.280 & 3.334 & 1.513 & 6350 & 4.21 & 3.500 \\
\hline 1.315 & 0.09 & 6250 & 3.333 & 0.023 & 0.694 & 0.283 & 2.9 & 0.00241 & 0.0871 & 1.292 & 3.330 & 1.561 & 6248 & 4.17 & 0.000 \\
\hline 1.310 & 0.09 & 6250 & 3.333 & 0.023 & 0.687 & 0.289 & 2.9 & 0.00248 & 0.0878 & 1.290 & 3.330 & 1.561 & 6248 & 4.17 & 1.500 \\
\hline 1.306 & 0.09 & 6250 & 3.333 & 0.023 & 0.683 & 0.294 & 2.9 & 0.00253 & 0.0882 & 1.288 & 3.330 & 1.561 & 6248 & 4.17 & 2.500 \\
\hline 1.303 & 0.09 & 6250 & 3.333 & 0.023 & 0.679 & 0.298 & 2.9 & 0.00258 & 0.0886 & 1.286 & 3.329 & 1.561 & 6248 & 4.17 & 3.500 \\
\hline 1.403 & 0.25 & 6250 & 3.333 & 0.033 & 0.684 & 0.283 & 2.2 & 0.00183 & 0.0815 & 1.313 & 3.335 & 1.561 & 6251 & 4.20 & 0.000 \\
\hline 1.384 & 0.25 & 6250 & 3.333 & 0.033 & 0.662 & 0.305 & 2.2 & 0.00207 & 0.0837 & 1.305 & 3.336 & 1.561 & 6251 & 4.19 & 1.500 \\
\hline 1.371 & 0.25 & 6250 & 3.333 & 0.033 & 0.647 & 0.320 & 2.3 & 0.00223 & 0.0851 & 1.299 & 3.337 & 1.561 & 6251 & 4.19 & 2.500 \\
\hline 1.358 & 0.25 & 6250 & 3.333 & 0.033 & 0.632 & 0.334 & 2.3 & 0.00240 & 0.0865 & 1.293 & 3.337 & 1.561 & 6251 & 4.18 & 3.500 \\
\hline 1.340 & 0.17 & 6250 & 3.122 & 0.028 & 0.689 & 0.283 & 2.5 & 0.00218 & 0.0821 & 1.259 & 3.127 & 1.511 & 6252 & 4.21 & 0.000 \\
\hline 1.329 & 0.17 & 6250 & 3.122 & 0.028 & 0.676 & 0.297 & 2.5 & 0.00237 & 0.0835 & 1.254 & 3.125 & 1.511 & 6251 & 4.20 & 1.500 \\
\hline 1.321 & 0.17 & 6250 & 3.122 & 0.028 & 0.667 & 0.306 & 2.6 & 0.00249 & 0.0845 & 1.250 & 3.124 & 1.511 & 6251 & 4.20 & 2.500 \\
\hline 1.313 & 0.17 & 6250 & 3.122 & 0.028 & 0.658 & 0.315 & 2.6 & 0.00261 & 0.0854 & 1.246 & 3.123 & 1.511 & 6250 & 4.20 & 3.500 \\
\hline 1.378 & 0.17 & 6250 & 3.558 & 0.028 & 0.689 & 0.283 & 2.5 & 0.00199 & 0.0861 & 1.350 & 3.563 & 1.613 & 6252 & 4.16 & 0.000 \\
\hline 1.367 & 0.17 & 6250 & 3.558 & 0.028 & 0.676 & 0.297 & 2.5 & 0.00214 & 0.0875 & 1.344 & 3.561 & 1.613 & 6251 & 4.16 & 1.500 \\
\hline 1.359 & 0.17 & 6250 & 3.558 & 0.028 & 0.667 & 0.306 & 2.5 & 0.00224 & 0.0885 & 1.340 & 3.560 & 1.613 & 6251 & 4.16 & 2.500 \\
\hline 1.351 & 0.17 & 6250 & 3.558 & 0.028 & 0.658 & 0.315 & 2.6 & 0.00235 & 0.0895 & 1.337 & 3.559 & 1.613 & 6250 & 4.15 & 3.500 \\
\hline
\end{tabular}


Table 10. Summary of Observational Data for $\rho^{1} \mathrm{Cnc}$

\begin{tabular}{ccccl}
\hline \hline$T_{\text {eff }}(\mathrm{K})$ & {$[\mathrm{Fe} / \mathrm{H}]($ solar $)$} & $\log g(\mathrm{cgs})$ & $v \sin i\left(\mathrm{~km} \mathrm{~s}^{-1}\right)$ & \multicolumn{1}{c}{ Reference } \\
\hline $5336 \pm 90$ & $0.40 \pm 0.07$ & $4.47 \pm 0.10$ & $2.5 \pm 1.0$ & Fuhrmann et al. 1998 \\
$5250 \pm 70$ & $0.45 \pm 0.03$ & $4.40 \pm 0.15$ & & Gonzalez 1998a \\
$5150 \pm 75$ & $0.29 \pm 0.06$ & $4.15 \pm 0.05$ & $1.4 \pm 0.5$ & Gonzalez 1997a \\
& & $1 \pm 1$ & Baliunas et al. 1997 \\
& $0.414 \pm 0.096$ & & Taylor 1991 \\
$5100 \pm 150$ & 0.20 & & Arribas \& Martinez-Roger 1989 \\
5350 & 0.44 & & & Campbell 1978 \\
5200 & $0.24 \pm 0.09$ & 4.5 & & Perrin 1977 \\
5140 & 0.30 & 4.4 & & Oinas 1977 \\
5196 & 0.11 & 4.4 & & Bakos 1971 \\
4460 & -0.15 & & & \\
\hline \hline
\end{tabular}

${ }^{\mathrm{d}}$ This value is based on a statistical analysis of previous measurements and not any new observations. 
Table 11. Stellar Models for $\rho^{1}$ Cnc

\begin{tabular}{|c|c|c|c|c|c|c|c|c|c|c|c|c|c|c|c|}
\hline $\begin{array}{c}M_{*} \\
\text { Solar }\end{array}$ & $\begin{array}{l}{\left[\frac{F e}{H}\right]} \\
\text { Solar }\end{array}$ & $\begin{array}{c}T_{\text {eff }} \\
\mathrm{K}\end{array}$ & $\begin{array}{c}L_{*} \\
\text { Solar }\end{array}$ & $\mathrm{Z}$ & $\mathrm{X}$ & $\mathrm{Y}$ & $\begin{array}{l}\text { Age } \\
\text { Gyr }\end{array}$ & $\begin{array}{c}M_{c e} \\
\text { Solar }\end{array}$ & $\begin{array}{l}\text { PSH } \\
\text { Solar }\end{array}$ & $\begin{array}{c}R_{c e} \\
\text { Solar }\end{array}$ & $\begin{array}{c}L_{*} \\
\text { Solar }\end{array}$ & $\begin{array}{c}R_{*} \\
\text { Solar }\end{array}$ & $\begin{array}{c}T_{\text {eff }} \\
\mathrm{K}\end{array}$ & $\begin{array}{c}\log g \\
\operatorname{cgs}\end{array}$ & $\begin{array}{c}\frac{\delta Y}{\delta Z} \\
\text { Solar }\end{array}$ \\
\hline 1.029 & 0.45 & 5300 & 0.608 & 0.053 & 0.613 & 0.334 & 2.4 & 0.04444 & 0.0798 & 0.647 & 0.612 & 0.927 & 5308 & 4.52 & 1.500 \\
\hline 0.951 & 0.45 & 5300 & 0.608 & 0.053 & 0.579 & 0.368 & 8.4 & 0.04583 & 0.0825 & 0.628 & 0.610 & 0.927 & 5305 & 4.48 & 2.500 \\
\hline 0.873 & 0.45 & 5300 & 0.608 & 0.053 & 0.544 & 0.403 & 14.3 & 0.04722 & 0.0853 & 0.609 & 0.609 & 0.927 & 5303 & 4.45 & 3.500 \\
\hline 1.048 & 0.39 & 5275 & 0.608 & 0.046 & 0.671 & 0.283 & 1.7 & 0.04703 & 0.0809 & 0.656 & 0.606 & 0.936 & 5270 & 4.52 & 0.000 \\
\hline 0.971 & 0.39 & 5275 & 0.608 & 0.046 & 0.630 & 0.324 & 7.9 & 0.04798 & 0.0835 & 0.637 & 0.608 & 0.936 & 5275 & 4.48 & 1.500 \\
\hline 0.920 & 0.39 & 5275 & 0.608 & 0.046 & 0.602 & 0.351 & 12.1 & 0.04862 & 0.0851 & 0.624 & 0.610 & 0.936 & 5279 & 4.46 & 2.500 \\
\hline 0.868 & 0.39 & 5275 & 0.608 & 0.046 & 0.575 & 0.379 & 16.3 & 0.04925 & 0.0868 & 0.611 & 0.611 & 0.936 & 5282 & 4.43 & 3.500 \\
\hline 0.978 & 0.45 & 5325 & 0.608 & 0.053 & 0.579 & 0.368 & 5.4 & 0.04404 & 0.0803 & 0.631 & 0.610 & 0.919 & 5329 & 4.50 & 2.500 \\
\hline 0.896 & 0.45 & 5325 & 0.608 & 0.053 & 0.544 & 0.403 & 11.6 & 0.04466 & 0.0829 & 0.613 & 0.611 & 0.919 & 5330 & 4.47 & 3.500 \\
\hline 1.006 & 0.42 & 5300 & 0.608 & 0.049 & 0.622 & 0.329 & 4.1 & 0.04541 & 0.0808 & 0.642 & 0.609 & 0.927 & 5302 & 4.51 & 1.500 \\
\hline 0.945 & 0.42 & 5300 & 0.608 & 0.049 & 0.591 & 0.360 & 8.9 & 0.04620 & 0.0828 & 0.627 & 0.609 & 0.927 & 5303 & 4.48 & 2.500 \\
\hline 0.885 & 0.42 & 5300 & 0.608 & 0.049 & 0.560 & 0.390 & 13.7 & 0.04699 & 0.0847 & 0.612 & 0.609 & 0.927 & 5303 & 4.45 & 3.500 \\
\hline 1.045 & 0.48 & 5300 & 0.608 & 0.057 & 0.603 & 0.340 & 1.2 & 0.04348 & 0.0792 & 0.651 & 0.616 & 0.928 & 5315 & 4.52 & 1.500 \\
\hline 0.959 & 0.48 & 5300 & 0.608 & 0.057 & 0.565 & 0.378 & 7.7 & 0.04580 & 0.0823 & 0.629 & 0.609 & 0.927 & 5303 & 4.48 & 2.500 \\
\hline 0.872 & 0.48 & 5300 & 0.608 & 0.057 & 0.527 & 0.416 & 14.2 & 0.04811 & 0.0854 & 0.607 & 0.603 & 0.927 & 5290 & 4.45 & 3.500 \\
\hline 0.991 & 0.45 & 5300 & 0.569 & 0.053 & 0.579 & 0.368 & 2.8 & 0.04437 & 0.0775 & 0.622 & 0.573 & 0.897 & 5308 & 4.53 & 2.500 \\
\hline 0.898 & 0.45 & 5300 & 0.569 & 0.053 & 0.544 & 0.403 & 10.1 & 0.04585 & 0.0807 & 0.601 & 0.568 & 0.897 & 5298 & 4.49 & 3.500 \\
\hline 1.020 & 0.45 & 5325 & 0.650 & 0.053 & 0.613 & 0.334 & 4.7 & 0.04403 & 0.0826 & 0.657 & 0.653 & 0.950 & 5330 & 4.49 & 1.500 \\
\hline 0.950 & 0.45 & 5325 & 0.650 & 0.053 & 0.579 & 0.368 & 9.7 & 0.04559 & 0.0850 & 0.640 & 0.650 & 0.950 & 5325 & 4.46 & 2.500 \\
\hline 0.881 & 0.45 & 5325 & 0.650 & 0.053 & 0.544 & 0.403 & 14.7 & 0.04714 & 0.0874 & 0.622 & 0.648 & 0.950 & 5320 & 4.43 & 3.500 \\
\hline
\end{tabular}


Table 12. Summary of Observational Data for $\rho$ CrB

\begin{tabular}{ccccl}
\hline \hline$T_{\text {eff }}(\mathrm{K})$ & {$[\mathrm{Fe} / \mathrm{H}]($ solar $)$} & $\log g(\mathrm{cgs})$ & $v \sin i\left(\mathrm{~km} \mathrm{~s}^{-1}\right)$ & \multicolumn{1}{c}{ Reference } \\
\hline $5821 \pm 80$ & $-0.24 \pm 0.08$ & $4.12 \pm 0.10$ & $1.0 \pm 1.0$ & Fuhrmann et al. 1998 \\
$5750 \pm 75$ & $-0.29 \pm 0.06$ & $4.10 \pm 0.05$ & $\sim 1.5$ & Gonzalez 1998 \\
& & 4.27 & & Kunzl et al. 1997 \\
5745 & -0.22 & 4.11 & & Gratton et al. 1996 \\
5905 & -0.24 & 4.20 & & Marsakov et al. 1995 \\
5782 & -0.26 & 4.24 & & Edvardsson et al. 1993b \\
& & & $1.5 \pm 1.0$ & Soderblom 1982 \\
5780 & -0.17 & 3.98 & & Hearnshaw 1974 \\
5860 & -0.14 & & & Alexander 1967 \\
5663 & -0.20 & & & Wallerstein 1962 \\
\hline \hline
\end{tabular}


Table 13. Stellar Models for $\rho \mathrm{CrB}$

\begin{tabular}{|c|c|c|c|c|c|c|c|c|c|c|c|c|c|c|c|}
\hline $\begin{array}{c}M_{*} \\
\text { Solar }\end{array}$ & $\begin{array}{l}{\left[\frac{F e}{H}\right]} \\
\text { Solar }\end{array}$ & $\begin{array}{c}T_{\text {eff }} \\
\mathrm{K}\end{array}$ & $\begin{array}{c}L_{*} \\
\text { Solar }\end{array}$ & $\mathrm{Z}$ & $\mathrm{X}$ & $\mathrm{Y}$ & $\begin{array}{l}\text { Age } \\
\text { Gyr }\end{array}$ & $\begin{array}{c}M_{c e} \\
\text { Solar }\end{array}$ & $\begin{array}{l}\text { PSH } \\
\text { Solar }\end{array}$ & $\begin{array}{c}R_{c e} \\
\text { Solar }\end{array}$ & $\begin{array}{c}L_{*} \\
\text { Solar }\end{array}$ & $\begin{array}{c}R_{*} \\
\text { Solar }\end{array}$ & $\begin{array}{c}T_{\text {eff }} \\
\mathrm{K}\end{array}$ & $\begin{array}{c}\log g \\
\operatorname{cgs}\end{array}$ & $\begin{array}{c}\frac{\delta Y}{\delta Z} \\
\text { Solar }\end{array}$ \\
\hline 0.874 & -0.23 & 5750 & 1.796 & 0.011 & 0.706 & 0.283 & 14.6 & 0.03400 & 0.1256 & 0.873 & 1.796 & 1.354 & 5750 & 4.12 & 0.000 \\
\hline 0.886 & -0.23 & 5750 & 1.796 & 0.011 & 0.718 & 0.271 & 14.3 & 0.03341 & 0.1250 & 0.879 & 1.796 & 1.354 & 5750 & 4.12 & 1.500 \\
\hline 0.894 & -0.23 & 5750 & 1.796 & 0.011 & 0.725 & 0.264 & 14.1 & 0.03301 & 0.1247 & 0.883 & 1.796 & 1.354 & 5750 & 4.13 & 2.500 \\
\hline 0.902 & -0.23 & 5750 & 1.796 & 0.011 & 0.733 & 0.256 & 14.0 & 0.03262 & 0.1243 & 0.887 & 1.795 & 1.354 & 5750 & 4.13 & 3.500 \\
\hline 0.863 & -0.23 & 5675 & 1.796 & 0.011 & 0.706 & 0.283 & 15.5 & 0.04391 & 0.1334 & 0.865 & 1.795 & 1.390 & 5675 & 4.09 & 0.000 \\
\hline 0.875 & -0.23 & 5675 & 1.796 & 0.011 & 0.718 & 0.271 & 15.3 & 0.04330 & 0.1330 & 0.871 & 1.795 & 1.390 & 5674 & 4.09 & 1.500 \\
\hline 0.883 & -0.23 & 5675 & 1.796 & 0.011 & 0.725 & 0.264 & 15.1 & 0.04290 & 0.1326 & 0.875 & 1.794 & 1.390 & 5674 & 4.10 & 2.500 \\
\hline 0.890 & -0.23 & 5675 & 1.796 & 0.011 & 0.733 & 0.256 & 15.0 & 0.04249 & 0.1323 & 0.879 & 1.794 & 1.390 & 5673 & 4.10 & 3.500 \\
\hline 0.888 & -0.23 & 5825 & 1.796 & 0.011 & 0.706 & 0.283 & 13.4 & 0.02581 & 0.1177 & 0.882 & 1.797 & 1.319 & 5825 & 4.15 & 0.000 \\
\hline 0.900 & -0.23 & 5825 & 1.796 & 0.011 & 0.718 & 0.271 & 13.2 & 0.02536 & 0.1171 & 0.887 & 1.796 & 1.319 & 5825 & 4.15 & 1.500 \\
\hline 0.909 & -0.23 & 5825 & 1.796 & 0.011 & 0.725 & 0.264 & 13.0 & 0.02507 & 0.1167 & 0.891 & 1.796 & 1.319 & 5825 & 4.16 & 2.500 \\
\hline 0.917 & -0.23 & 5825 & 1.796 & 0.011 & 0.733 & 0.256 & 12.8 & 0.02477 & 0.1163 & 0.895 & 1.796 & 1.319 & 5825 & 4.16 & 3.500 \\
\hline 0.896 & -0.17 & 5750 & 1.796 & 0.013 & 0.704 & 0.283 & 13.8 & 0.03231 & 0.1242 & 0.884 & 1.796 & 1.354 & 5750 & 4.13 & 0.000 \\
\hline 0.906 & -0.17 & 5750 & 1.796 & 0.013 & 0.713 & 0.274 & 13.6 & 0.03191 & 0.1237 & 0.888 & 1.796 & 1.354 & 5750 & 4.13 & 1.500 \\
\hline 0.912 & -0.17 & 5750 & 1.796 & 0.013 & 0.720 & 0.268 & 13.5 & 0.03163 & 0.1234 & 0.891 & 1.796 & 1.354 & 5750 & 4.13 & 2.500 \\
\hline 0.919 & -0.17 & 5750 & 1.796 & 0.013 & 0.726 & 0.262 & 13.4 & 0.03136 & 0.1231 & 0.894 & 1.796 & 1.354 & 5750 & 4.14 & 3.500 \\
\hline 0.862 & -0.23 & 5750 & 1.673 & 0.011 & 0.706 & 0.283 & 15.3 & 0.03306 & 0.1208 & 0.846 & 1.673 & 1.307 & 5750 & 4.14 & 0.000 \\
\hline 0.875 & -0.23 & 5750 & 1.673 & 0.011 & 0.718 & 0.271 & 15.0 & 0.03258 & 0.1203 & 0.852 & 1.673 & 1.307 & 5750 & 4.15 & 1.500 \\
\hline 0.883 & -0.23 & 5750 & 1.673 & 0.011 & 0.725 & 0.264 & 14.8 & 0.03226 & 0.1200 & 0.855 & 1.673 & 1.307 & 5750 & 4.15 & 2.500 \\
\hline 0.891 & -0.23 & 5750 & 1.673 & 0.011 & 0.733 & 0.256 & 14.6 & 0.03194 & 0.1197 & 0.859 & 1.673 & 1.307 & 5750 & 4.15 & 3.500 \\
\hline 0.887 & -0.23 & 5750 & 1.928 & 0.011 & 0.706 & 0.283 & 13.8 & 0.03464 & 0.1303 & 0.903 & 1.928 & 1.403 & 5750 & 4.09 & 0.000 \\
\hline 0.899 & -0.23 & 5750 & 1.928 & 0.011 & 0.718 & 0.271 & 13.6 & 0.03403 & 0.1297 & 0.909 & 1.928 & 1.403 & 5750 & 4.10 & 1.500 \\
\hline 0.907 & -0.23 & 5750 & 1.928 & 0.011 & 0.725 & 0.264 & 13.4 & 0.03362 & 0.1293 & 0.913 & 1.928 & 1.403 & 5750 & 4.10 & 2.500 \\
\hline 0.915 & -0.23 & 5750 & 1.928 & 0.011 & 0.733 & 0.256 & 13.3 & 0.03321 & 0.1289 & 0.917 & 1.928 & 1.403 & 5750 & 4.11 & 3.500 \\
\hline
\end{tabular}




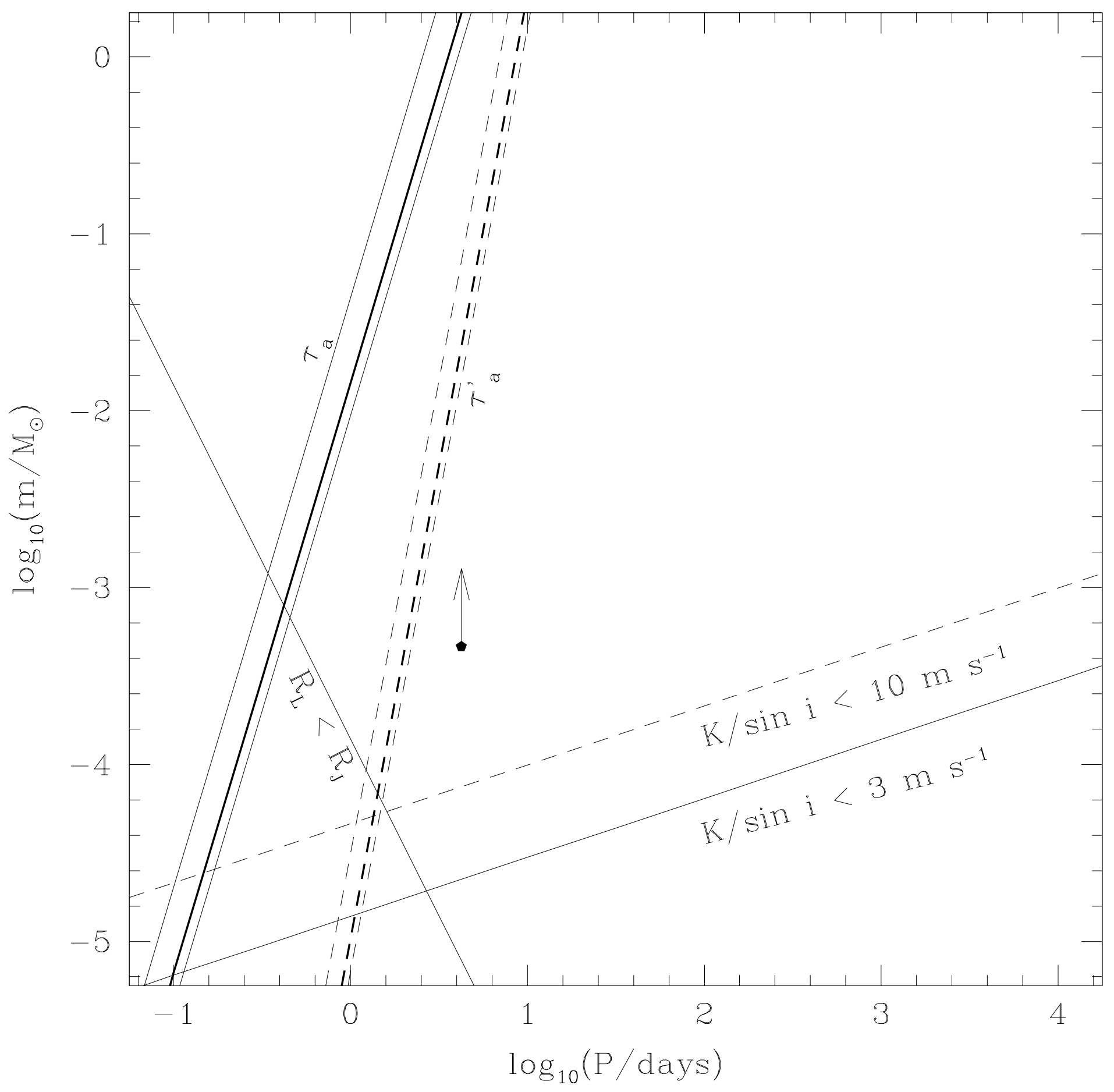




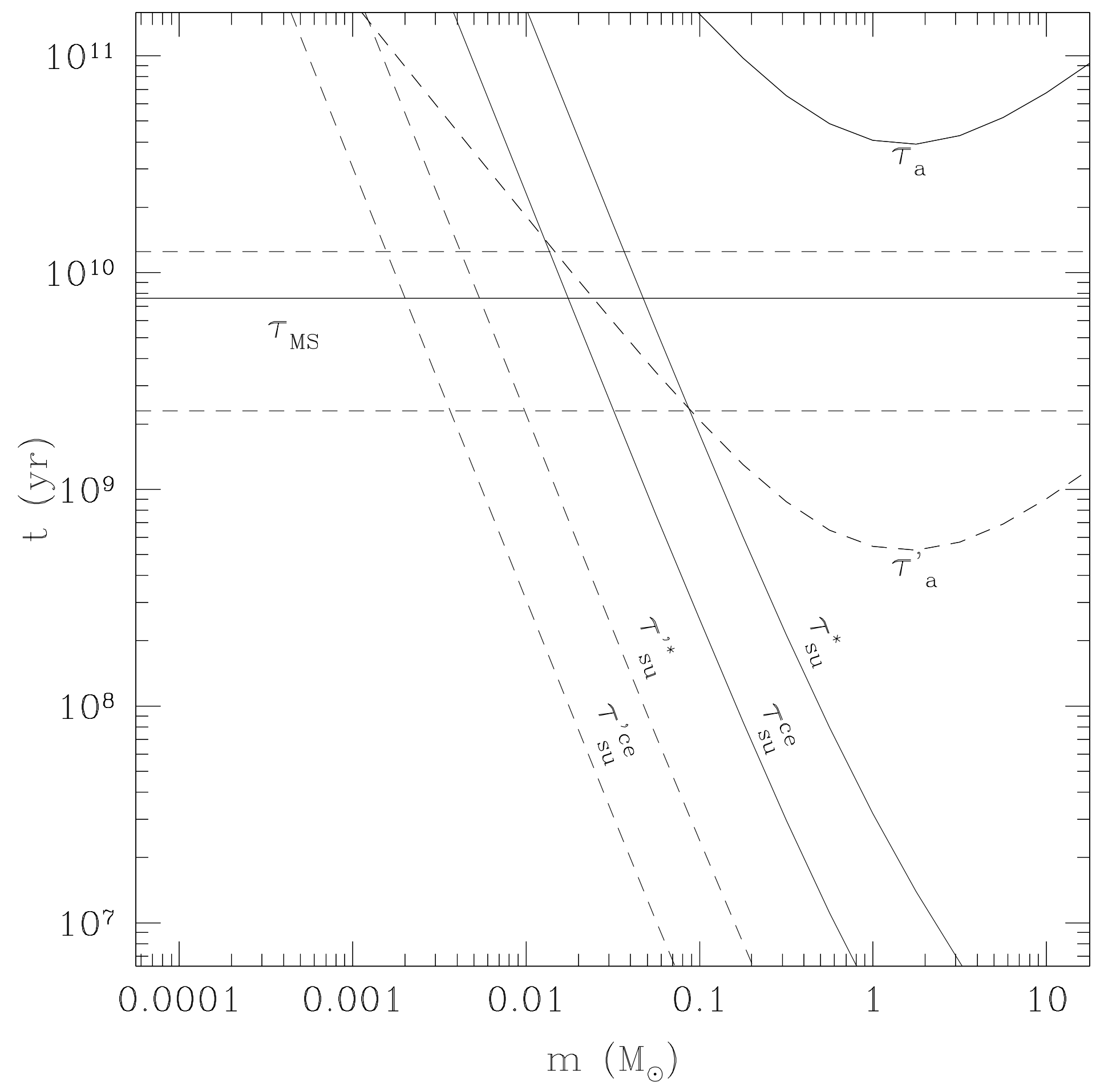




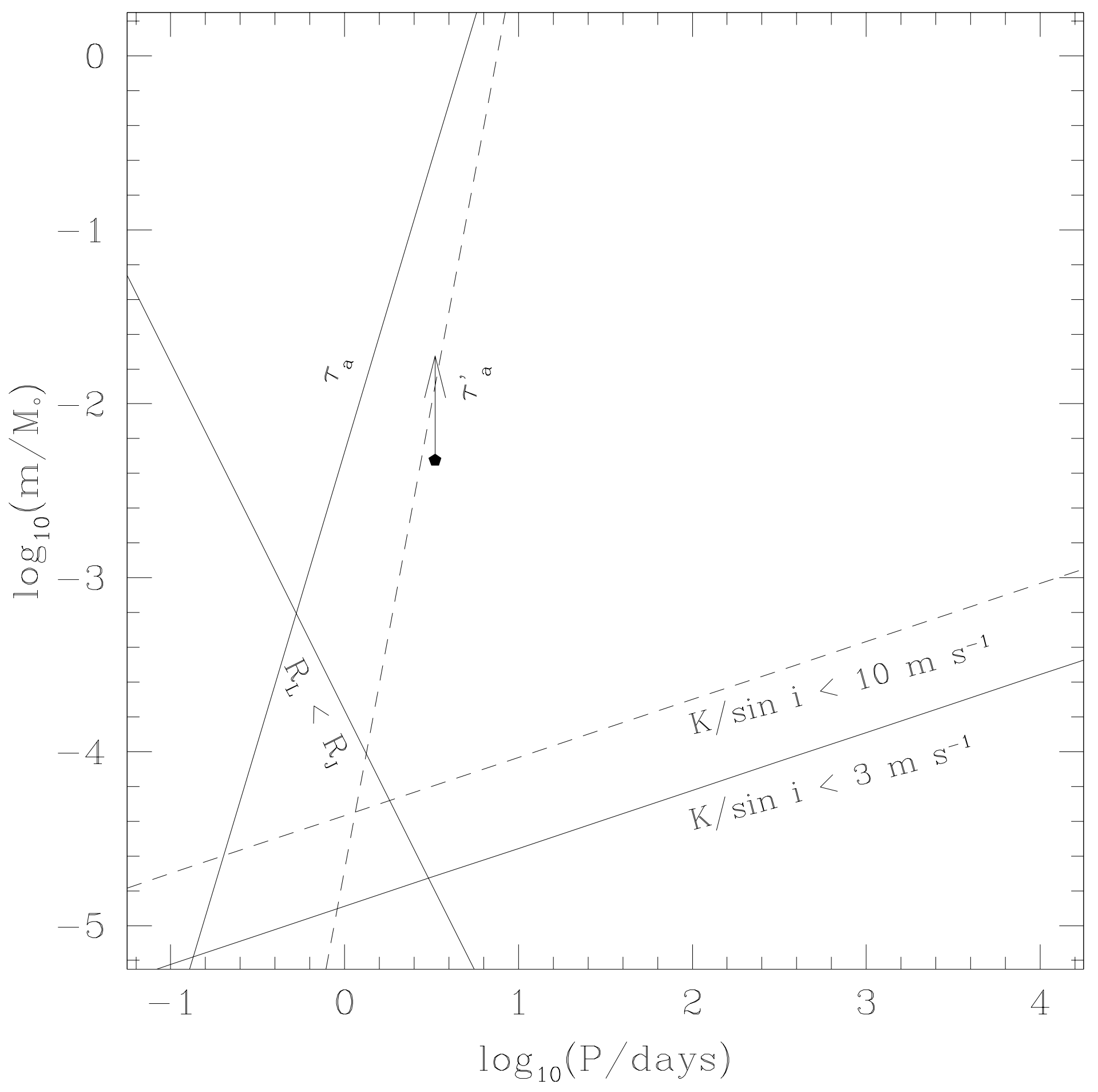




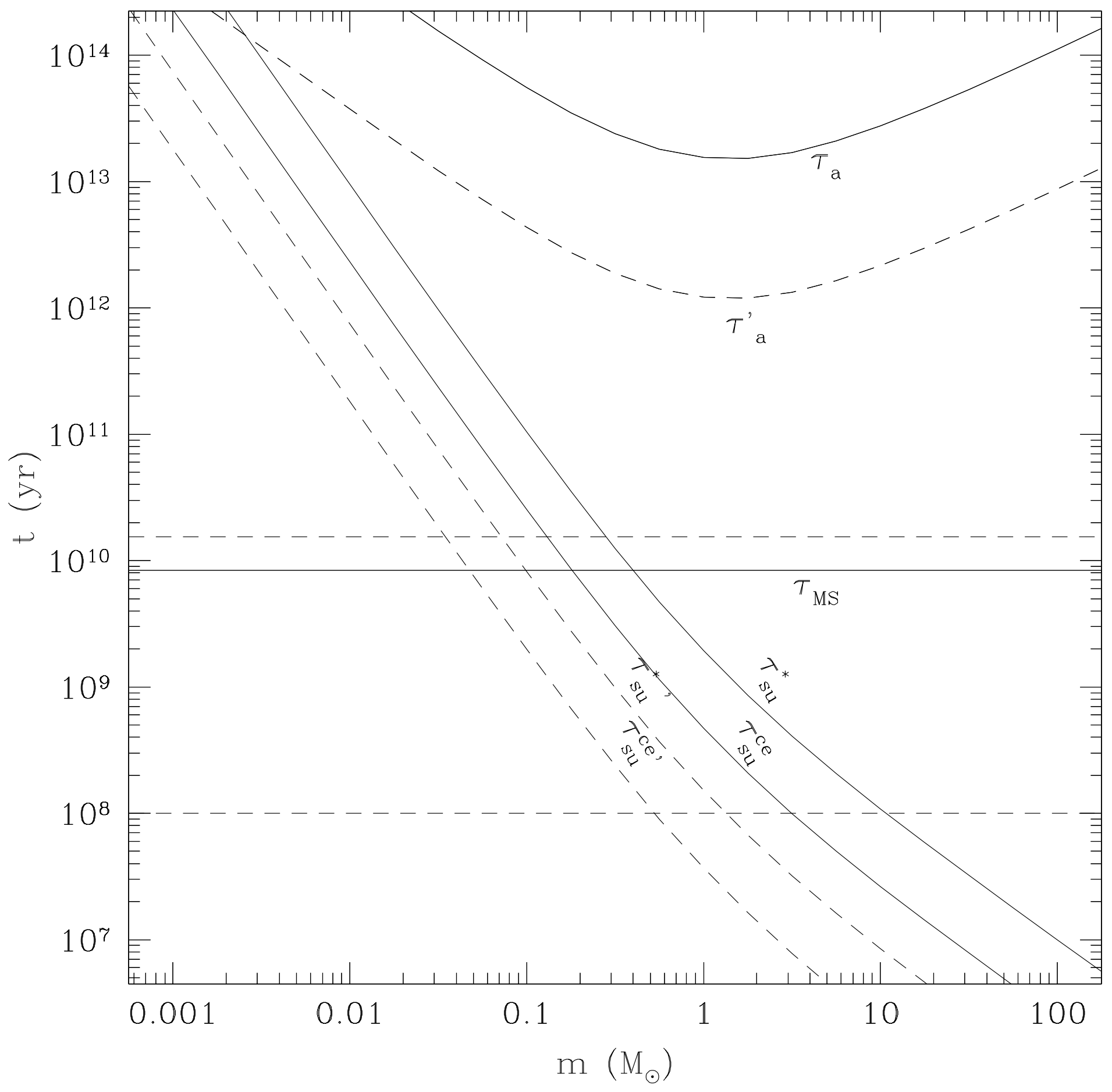




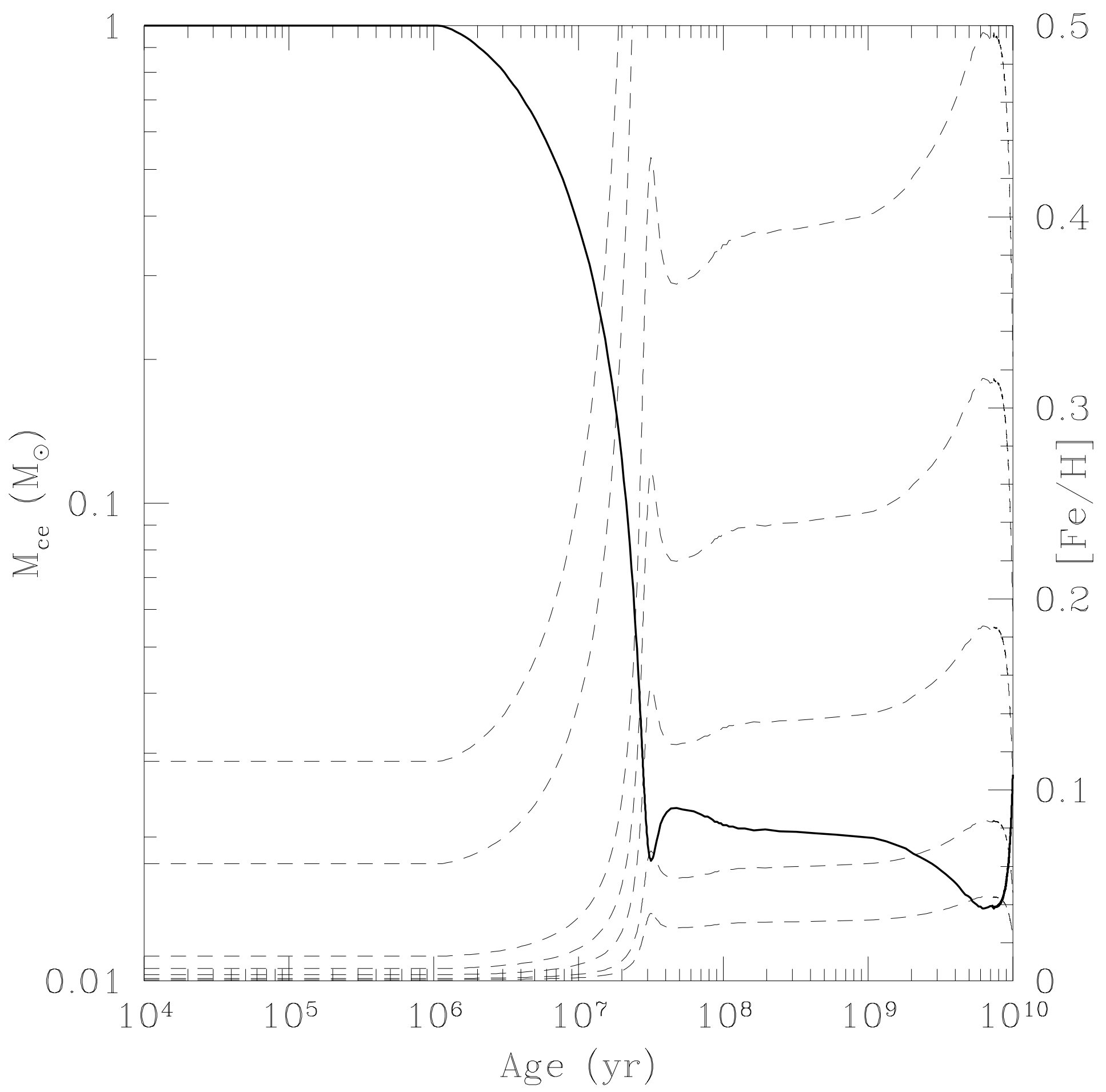

